\title{
Critical parameters for design and development of multivalent nanoconstructs: recent trends
}

\author{
Avijit Kumar Bakshi ${ }^{1} \cdot$ Tanweer Haider $^{1}\left[\mathbb{C} \cdot\right.$ Rahul Tiwari $^{1}\left[\mathbb{D} \cdot\right.$ Vandana Soni $^{1} \mathbb{C}$
}

Accepted: 30 November 2021 / Published online: 11 January 2022

(c) Controlled Release Society 2021

\begin{abstract}
A century ago, the groundbreaking concept of the magic bullet was given by Paul Ehrlich. Since then, this concept has been extensively explored in various forms to date. The concept of multivalency is among such advancements of the magic bullet concept. Biologically, the concept of multivalency plays a critical role in significantly huge numbers of biochemical interactions. This concept is the sole reason behind the higher affinity of biological molecules like viruses to more selectively target the host cell surface receptors. Multivalent nanoconstructs are a promising approach for drug delivery by the active targeting principle. Designing and developing effective and target-specific multivalent drug delivery nanoconstructs, on the other hand, remain a challenge. The underlying reason for this is a lack of understanding of the crucial interactions between ligands and cell surface receptors, as well as the design of nanoconstructs. This review highlights the need for a better theoretical understanding of the multivalent effect of what happens to the receptor-ligand complex after it has been established. Furthermore, the critical parameters for designing and developing robust multivalent systems have been emphasized. We have also discussed current advances in the design and development of multivalent nanoconstructs for drug delivery. We believe that a thorough knowledge of theoretical concepts and experimental methodologies may transform a brilliant idea into clinical translation.
\end{abstract}

Keywords Multivalency · Multivalent nanoconstructs · Drug delivery $\cdot$ Quantification · Design and development

\section{Introduction}

A multivalent ligand may be defined as a system that consists of multiple ligand moieties, which can bind to more than one binding site or receptors accessible on the target site. A prominent example is a multivalent interaction between a virus and its target cells, which results in initially strong adhesion. The notion of ligand multivalency arose from or was inspired by the multivalent interaction properties present in microorganisms. Monovalent drugs are the most common clinical approach in medicine. Because pathophysiological alterations caused by complex/adopted microorganisms or cancer cells have such a strong affinity for multivalent cell surface receptors, monovalent medicines must be employed in very high doses. As a result, designing and manufacturing a multivalent drug delivery scaffold have

Vandana Soni

vsoni@dhsgsu.edu.in; drvandanasoni@gmail.com

1 Department of Pharmaceutical Sciences, Dr. Harisingh Gour University, Madhya Pradesh, Sagar 470003, India immense potential for effectively combating viruses and bacteria, as well as regulating cancer cell development [1].

The most typical multivalent in vivo recognition phenomena involve several copies of cell-surface receptors engaging with multivalent ligands. Many biochemical processes, including molecular recognition and signal transduction initiation, which includes many interactions between ligands and their receptors [2], as well as other immune functions, such as cellular attachment by infectious agents, antibody recognition, pathogen opsonization, and immune-inflammatory response, rely on multivalent interaction [2-5]. These multivalent interactions have unique effects, such as the advantage of several binding events that enhance binding on a molecular level in ways that monovalent structures do not. Multivalent systems have many significant benefits that make them a potential therapeutic option [4]. To begin with, multivalent agents may significantly reduce pathogen cell adherence by using a dynamic process involving steric stabilization that traditional therapeutic approaches are unable to regulate. Second, biological interactions between receptors and ligands are often modest. By increasing functional 
avidity, multivalency may overcome these limitations. Multivalent interactions are distinguished from monovalent interactions by their high affinity and relatively slow dissociation kinetics. Third, multivalent compounds have the ability to cluster receptors and modulate signal transduction pathways. Fourth, the attachment of several targeting groups and therapeutic agents to a single mechanism permits these mechanisms to be targeted to multiple receptors at the same time. Fifth, the mechanical features of existing therapeutic agents, such as their prolonged duration in the systemic circulation, may be improved by manipulating the properties of multivalent nanostructures [4]. Furthermore, multivalent structures would generate matrices that might regulate some mechanisms in several contexts, something that typical monovalent nanocarrier-mediated therapies are unable to do. The molecular mechanisms by which multivalent ligands carry out their activities determine their efficacy. A detailed knowledge of the inputs that distinct mechanisms may give for multivalent ligand-receptor recognition is required to develop an effective, powerful, and selective multivalent drug delivery system [4].

The incorporation of the influenza virus into the cell membrane, antibody-mediated activation of the complement cascade, migration of immunological cells into the inflammatory area, stimulation of the $\mathrm{T}$ cell, attachment of immunoglobulin (Ig) E molecules to macrophages or basophils for the formation of $\operatorname{IgE}$ antibodies [6, 7], and the binding of Shiga toxin to cell membranes [8-11] are all examples of multivalent processes that cause pathological phenomena. Multivalent ligands may also increase L-selectin clustering on the surface of leukocytes, resulting in glycoprotein shedding [12]. Furthermore, multidentate sucrose ligand substitutes demonstrate avidity, or the sum of many binding interactions that occur when multiple ligand/ receptor complexes are triggered, as well as improved accuracy in protein-carbohydrate recognition processes [13-16]. The simultaneous binding of multiple ligands to receptors enhances ligand affinity, strength, and stability as compared to free monovalent ligands. The binding of ligands to their receptor molecules is improved much further by multivalent interaction. Many pathophysiologies, including hepatitis, influenza, tuberculosis, Alzheimer's disease, Huntington's disease, diabetes, and cancer, rely on multivalency [17].

Multivalent ligands may be able to address problems that typical monovalent materials cannot due to their several the recognition feature. For molecular identification, simulation, diagnosis, and treatment of diseases, the use of multivalent ligands in the design and development of drugs has been widely investigated. Multivalent vaccines, for example, have been utilized by explicitly priming CD $8+$ and CD4 + T-specific tumor cells in cancer treatment. The ability to induce dimerization or oligomerization of the receptors on the cell surface determines the effectiveness of a multivalent vaccine [18-20]. Conjugation of multiple copies of targeting ligands, drugs, and dyes has also become a frequent strategy for developing theranostics resources that may be used for disease diagnosis and treatment. To develop a multivalent theranostics system useful in detecting and treating diseases, several copies of targeting ligands and imaging agents may be conjugated to polymer scaffolds containing potential therapeutic molecules. Various aspects of multivalency will be discussed in this review in order to develop a robust multivalent drug delivery system.

\section{Design and development of multivalent targeted nanosystems}

For the unique binding of related receptors to therapeutic purposes, several multivalent ligands have been developed. Multivalency in drug delivery systems can also be made possible by binding many copies of ligands to nanocarriers, such as polymer composites, dendrimers, micelles, and nanoparticles [21]. It is necessary to rationally formulate a multivalent ligand framework that is dependent on the ligand's intrinsic affinity, distance, and relative spatial orientation in order to use multivalency as a principle of organization and active involvement. The size and shape of the carrier system, the corresponding valency of the system, the ligand density and configuration, the size and flexibility of the linker, and the molecular structure and functional groups present in the linker are all parameters that are defined primarily by the choice of a multivalent carrier system [21]. These complex parameters should be considered when constructing a multivalent ligand grafted carrier system. This section addresses at the complex relationships that exist in the design and development of multivalent carrier systems.

\section{Dimensions of the nanocarrier}

Viruses and bacteria have evolved into a variety of precise sizes and shapes in order to properly mediate their interactions with target cells. Similarly, we must first understand how the dimensions (size and shape) of a synthetic nanocarrier impact its interaction with the biological system in order to design nanostructures capable of traversing the systemic circulation and targeting diseased cells. The cellular adhesion and interaction of the multivalent ligands on the nanocarrier are directly influenced by the nanocarrier size. The small and compact size of the scaffolds is considered ideal for targeted drug delivery, which may penetrate and propagate through membranes, or may be aggregated at tumor sites via a renowned process known as enhanced permeation and retention effect [22-24]. The well-defined shape and composition of the ligand allow it to be located at the target receptors. To achieve optimal binding performance, 
the scaffold's dimension must be tailored to accommodate its preferred accumulation at the target cell. Papp et al. [25] demonstrated that multivalent sialic acid-conjugated polymeric nanoparticles with particle sizes ranging from 50 to $100 \mathrm{~nm}$ (corresponding to the size range of influenza A virus) are significantly more effective at preventing influenza A virus binding than smaller guided nanoparticles. Similarly, Sykes et al. [26] investigated the influence of particle size on active targeting of transferrin conjugated gold nanoparticles and found that $60 \mathrm{~nm}$ gold nanoparticles are capable of rapid and enhanced tumor uptake. Propper tailoring of scaffold dimension is not possible with several scaffolds such as adamantine [27], calixarene [28], and cyclodextrin [29]. Because only a few numbers of ligands may be linked to these scaffold subtypes, they have low binding affinity for target proteins or cell surface receptors. Dendrimers, on the other hand, are an excellent example of a multivalent system that is tailored optimally for the desired dimension. They enable the formation of a branching network-like structure that allows multiple functional groups or ligands to be attached, hence potentiating the binding affinity. This potentiation of binding affinity might be owing to the steric impact of the backbone molecules, which is caused by the scaffold's increased size [30]. Multivalent ligands' binding patterns are also influenced by scaffold subtypes. Intramolecular binding may occur when multivalent ligands are grafted onto two-dimensional structures. Multivalent ligands grafted on a three-dimensional structure with the help of a linker, such as nanoparticles or dendrimers, on the other hand, are susceptible to intermolecular binding [29, 31].

In the context of biological systems, antibodies are likely the most extensively utilized materials for the study of multivalency, owing to the quaternary structure of antibody molecules that may interact with numerous recognition sites. Hence, these biological molecules are naturally equipped to provide enhanced binding affinity. Various antibodies, such as $\operatorname{IgE}, \operatorname{IgG}$, and $\operatorname{IgD}$, depict the monomeric antibody with two antigen-binding sites. Whereas IgA antibody has a dimeric form and provides four binding sites, IgM is the pentameric antibody with ten binding sites. As various antibodies represent distinct dimensions, their biological activity involving binding affinity also gets influenced [31]. Cremers et al. [32] have utilized antibody surface grafted DNA nanostructures to demonstrate that although the inherent affinity of targeting antibody remains unaltered, the number of antibody-receptor interactions remains lower than soluble antibodies governed by nanoconstruct's size and orientation. Based on these observations, the authors concluded that larger DNA nanostructures may cause steric hindrance and are the primary cause of reduced receptor binding potential. In a recent study, Choo et al. [33] have demonstrated that the dimensions of nanoconstructs impact active targeting. The authors compared aptamer AS1411 surface grafted gold nanostar and gold nanospheres $(50 \mathrm{~nm})$ on cells with and without nucleolin receptor expression using single-particle monitoring of translational and rotational motion. Despite possessing identical protein corona profiles, gold nanostars migrated faster under directed diffusion, according to the authors. Furthermore, in nucleolin-inhibited cells, gold nanostars showed faster rotational dynamics throughout smaller translational areas, but gold nanospheres showed no significant changes in translation. These variations in translational and rotational motions imply that the dimension of the nanoconstruct determines how the targeting moiety attaches to the cell membrane receptors. As a result, proper tailoring of a multivalent carrier system or scaffold is an important element to consider. It should be critically considered when designing a multivalent system.

\section{Valency of ligands on multivalent scaffolds}

The number of identical ligands grafted on a nanocarrier or scaffold with multivalent ligands is referred to as valency. In other words, valency refers to the number of various interactions of the same type that may occur through the host (cell surface receptors)-guest (ligand) interactions with entities that have complimentary features (Fig. 1).

The binding avidity and target specificity of a multivalent ligand improve dramatically as the valence of the ligand on the multivalent scaffold rises. To design an effective multivalent construct, this parameter must be severely optimized. Because they are less immunogenic and more stable in the systemic circulation, bivalent and trivalent antibody molecules are much more suitable for tumor targeting. Another key benefit is that they may be manufactured on a large scale [34]. The use of an antibody with a larger magnitude of valency is limited by molecular instability, which adversely affects its therapeutic potential [34].

The effect of valence on the performance of the multivalent system can be assessed by developing polymer composites of specific sizes. The valency of ligands associated with synthetic scaffolds or nanocarriers can be tailored consistently by adjusting the length or diameter of the scaffold or nanocarrier. The effect of dendrimer valency on performance may be examined in this context by analyzing various generations of dendrimers [35]. For instance, generation zero PAMAM dendrimers have a size of $1.5 \mathrm{~nm}$ and display a tetravalent system [35]. Every consecutive generation increases the diameter by 0.7 to $1.6 \mathrm{~nm}$, doubling the ligand valency. Kiessling et al. [36] also established the influence of ligand valency on the performance of the multivalent system (in terms of receptor binding and endocytosis). Dendrimers with increasing ligand (lactose residues) valency were synthesized in this work. The activity of these multivalent dendrimers was assessed by employing a protein panel (having different no. of saccharide receptors as binding sites). 
Fig. 1 Valency of the hostguest (ligand-receptor) complex

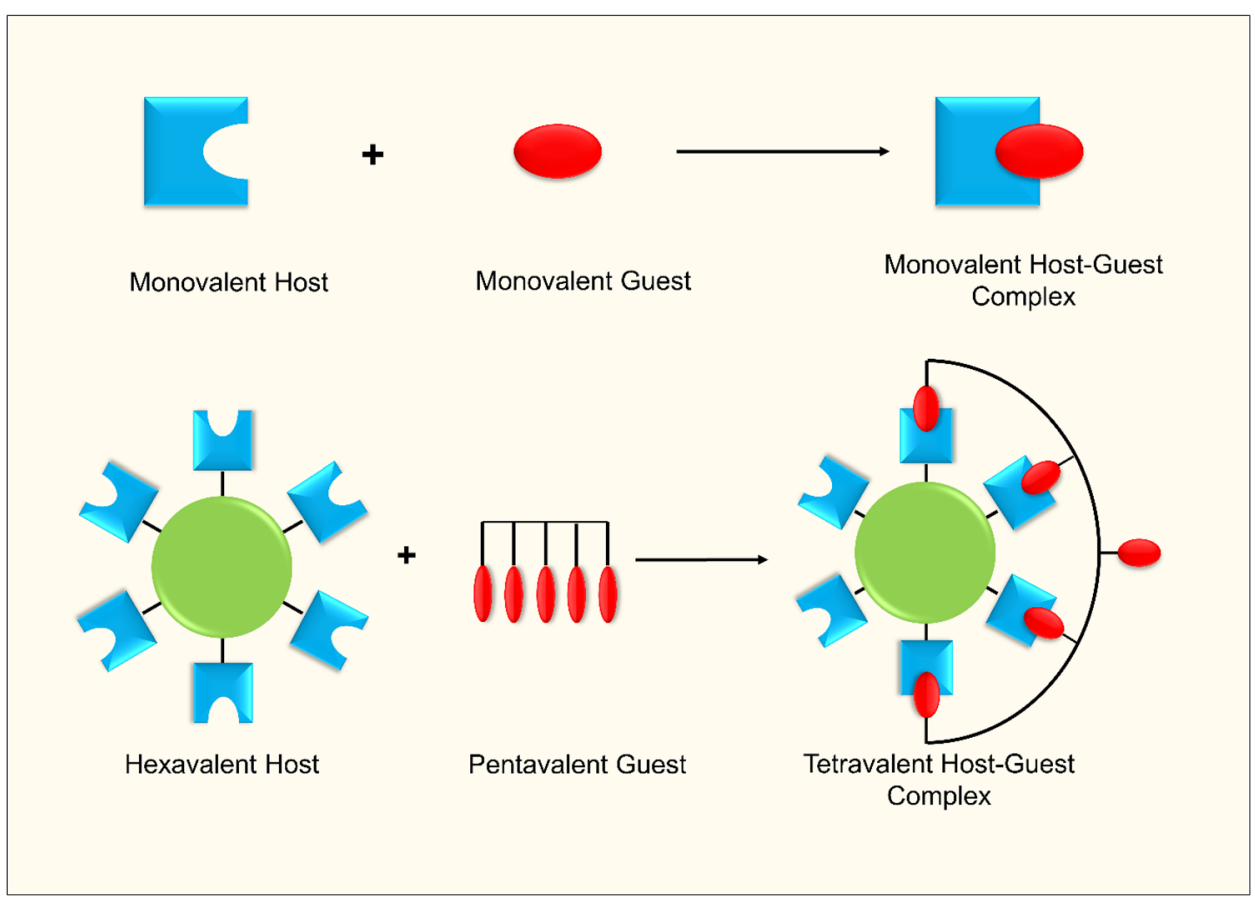

The performance of the multivalent dendrimer was assessed based on a number of binding interactions between ligand and saccharide residues, and the authors demonstrated that as dendrimer valency increases, so does the protein dendrimer interaction. The authors have also demonstrated that the magnitude of binding interaction depends on the dimensions of the target protein [36]. The need of properly tailoring the valency of ligands associated with multivalent systems to the target cell surface receptor is emphasized in this work.

Several polymerization reactions allow for precise monitoring of the ligand valency on the multivalent scaffold [37]. Precise polymerization reactions allow the pharmacologically active ligand to be produced with optimized valency [24]. Ring-opening metathesis reaction is a polymerization reaction that generates polymers, which are used as scaffolds for pharmacologically active multivalent ligands [38, 39]. The ruthenium-carbene catalysis reaction, which is mediated by ring-opening metathesis polymerization, may produce scaffolds with altered valency [40, 41]. Such ligands have been constructed to work as inhibitors of saccharideprotein interactions [42, 43], as ligands for the suppression of vancomycin-resistant bacteria [44], and as modulators of biochemical reactions $[45,46]$. Pertinently, the polymerization process accounts for precise monitoring of the multivalent ligand display and the ligand valency [37].

A multivalent peptide construct was developed by Cochran et al., incorporating peptide obtained from cytochrome $\mathrm{c}$ and complexing it with major histocompatibility complex (MHC). The resultant cytochrome c-MHC complex was biotinylated separately and combined with streptavidin to generate a tetravalent complex capable of engaging the $\mathrm{T}$ cell receptor. The quantity of effective cytochrome c-MHC complex in the assembly forming reaction was varied to promote monomeric, dimeric, trimeric, or tetrameric construct. The effects of the assemblies on $\mathrm{T}$ cell activation, extracellular $\mathrm{pH}$ alterations, and intracellular $\mathrm{Ca}^{2+}$ concentrations were assessed. In both cases, the tetramer was the sole ligand that induce a substantial rise in activity. These experimental data suggest that valency alterations have a significant impact on $\mathrm{T}$ cell activation. Similar results were achieved using chemically established peptide-based multivalent ligands; however, only the dimeric ligand was adequate to enable T cell-mediated signal transmission $[47,48]$.

This information recorded collectively demonstrates that valency is an essential feature of multivalent ligands necessary to enhance T cell-mediated transmissions. Many researchers exploited synthetic multivalent ligands to understand ligand valency's impact on B cell-mediated responses $[49,50]$. Several multivalent ligands based on dextran, polyvinyl alcohol, carboxymethylcellulose, and polyacrylamide have been constructed to check whether T cell valence antigens affect immune response [51]. Rodents were given such ligands, and the efficacy of immune responses was quantified. The immunological response elicited by the multivalent ligands was solely dependent on the ligand's valency in each instance, independent of the scaffold's composition or the level of polymer branching. Based on these findings, the authors hypothesize that the valence of ligands associated 
with scaffolds induces B cell receptor clustering and plays a significant role in determining function.

Radford et al. [52] generated an array of polymer-based affibody conjugates with valency varying from 2 to 10 peptides per polymer by conjugating an HPMA copolymer-based carrier system with HER-2 binding affibody peptide. The targeting ligands might crosslink the HER2 receptors and override the receptor's inherent "internalization-resistant" function, allowing the carrier to be internalized quickly and then trafficked to the lysosomes. According to the authors, this design resulted in considerably greater absorption kinetics and overall levels of intracellular dispersion than either an untargeted carrier system or a monovalent peptide (with over $90 \%$ of the surface-bound conjugate internalized within four hours). Furthermore, this enhancement was sensitive to a higher amount of surface grafting, which authors have demonstrated, reduces the conjugates' ability to crosslink receptors. The data strongly suggests that this is an inherent feature resulting from the multivalent conjugate design. Even at picomolar treatment doses, targeted conjugates exhibited high intracellular delivery. Untargeted HPMA copolymers, on the other hand, needed 1000-fold greater treatment concentrations to reach equivalent levels of intracellular accumulation, indicating the design's prospective relevance for increased intracellular drug delivery [52].

\section{The flexibility and length of the linker}

Entropic transition is the foremost hurdle to be conquered before intramolecular binding may occur. The binding event of a ligand grafted on the multivalent construct with the receptor hinders the movement and binding of the neighboring ligand during the multivalent interaction between the multivalent construct and the target receptors, undesirable in an unwanted entropic penalty [5].

Rigid linkers with restricted flexibility obstruct multivalent ligand orientation and efficient interactions with receptors. Flexible linkers, when attached to a multivalent ligand, may be implemented in a variety of configurations and orientations within the system to bind to target receptors with lower steric strain [5]. Earlier, Krishnamurthy et al. [53] and Shewmake et al. [54] demonstrated that the use of flexible linkers larger than the spacing between the neighboring cell surface receptors accounts for the potentiated binding event between the ligands and the receptors, significantly minimizing the conformational entropy. In a study by Hsu et al. [55], sulfated galactose combined with thrombin-binding aptamer-conjugated gold nanoparticles demonstrated extreme anticoagulant behavior against thrombin. The researchers addressed the essential role of extended and flexible thrombin binding aptamer in regulating the multivalent interactions with thrombin. However, a too flexible linker may minimize ligand avidity due to higher conformational entropy [5]. Several investigations [56-59] have supported this claim and provided light on the critical importance of conformational entropy loss associated with linkers. To minimize conformational entropy loss during ligand-receptor interaction, the linker moiety associated with multivalent ligand should be optimally rigid. This is because the more flexible the linker is, the more entropy it may lose owing to more accessible conformational states.

Along with this, the length of the linker that attaches the ligands to the scaffold must be critically optimized to avoid unwanted steric hindrance between the ligands, to provide enhanced spatial flexibility and binding avidity, and to ensure that the multivalent system achieves the desired pharmacological activity and stability in systemic circulation [21]. The linker that is far too long can result in undesirable conformation and render the multivalent ligand inappropriate for cellular binding [60]. Shiokawa et al. [61] revealed that moderately lengthy linkers may play an important role in giving stability to the multivalent construct and can greatly lengthen the half-life of a drug that is encapsulated or conjugated with the multivalent construct. Arnold et al. [62] demonstrated that the optimal length of the linker between ligands and the backbone of the multivalent construct, which basically produces integrin clustering as a result of multivalent activity, was $25-73 \mathrm{~nm}$. According to comparable research by Rosca et al. [63], the optimal length of the PEG linker that might produce multivalent interactions between ligands and integrins is $30 \mathrm{~nm}$. Hence, tailoring of both the parameters (length and flexibility of linker) should be considered for the proper design of the multivalent construct.

To summarize this section, linkers attached to multivalent ligands are critical players in defining the entropy associated with multivalent ligand-receptor interaction. Assume the linker is too short or too rigid. In such situation, the multivalent binding interaction between the ligand and receptor may be associated with a significant amount of steric strain, which may contribute to a decrease in the overall system's conformational entropy. Linkers that are unconditionally long and excessively flexible, on the other hand, have an excess of entropy. The stated linker may access multiple conformational states or be directed in various directions, resulting in a loss of the conformational entropy of the system, consequently reducing the probability of the ligand-receptor binding event. Ideally, the linker should be of optimal length and rigidity to make it easier for several multivalent ligands to engage several receptors simultaneously.

\section{Ligand density on the multivalent constructs}

The nature and degree of multivalent ligand interactions are proportional to the ligand density of the multivalent construct [64]. The ligand density is the number of ligand molecules in proportion to the number of side chains or functional 
groups of multivalent constructs that the ligand has bound. Gestwicki et al. [65] used particle-mediated clustering of target receptors before the immune response to demonstrate correlations between immunological response and multivalency. They demonstrated that the ligand density associated with the multivalent construct is a critical factor in mediating the receptors' formation of subsequent cluster forms, which play a role in signal transduction. High ligand density induces multivalent binding through the clustering effect between the receptors [66]. The pharmacological activity of ligand molecules may also be controlled by changing the ligand density on the multivalent construct [21]. The importance of the target ligand density in the development of the vaccine delivery mechanism has been exemplified. The ligand density of multivalent monoclonal antibodies to dendritic cell lectin nanoparticles loaded with antigens has influenced dendritic cell activity and corresponding $\mathrm{T}$ cell expressions. The amount of cytokine released by dendritic cells and $\mathrm{T}$ cells enhanced significantly due to the clustering of the target receptor with the optimum ligand density of monoclonal antibodies on the surface of the nanoparticles
[67]. We strongly recommend that interested readers read the study by Alkilany et al. [68]; with this in mind, it is critically necessary to take into consideration the active role of ligand density on multivalently targeted drug delivery nanoconstructs.

\section{Modes of interaction between multivalent ligands and the target receptors}

The extent of effect provided by the multivalent ligand constructs solely depends on the mechanism by which they interact with the target receptors. Generally, conventional monovalent ligands only account for the interaction with a single receptor molecule and cannot induce dimers and oligomers' formation. However, in the absence of multivalent ligands, it may efficiently trigger cluster formation inside the receptors. Researchers have suggested that multivalent ligands interact with the target receptors by exploiting five possible mechanisms [24, 64]. Figure 2 depicts a diagrammatic depiction of these interactions.
Fig. 2 Mechanisms by which multivalent ligands can interact with cell-surface receptors. a Multivalent ligands can bind oligomeric receptors by occupying multiple binding sites (chelate effect). b Multivalent ligands can cause receptors to cluster on the cell surface and may activate signaling pathways. c Multivalent ligands can occupy primary and secondary binding sites on a receptor. $\mathbf{d}$ Multivalent ligands display higher local concentrations of binding epitopes, which can result in higher apparent affinities (republished from Kiessling et al. [24] with permission from Elsevier, copyright 2020)

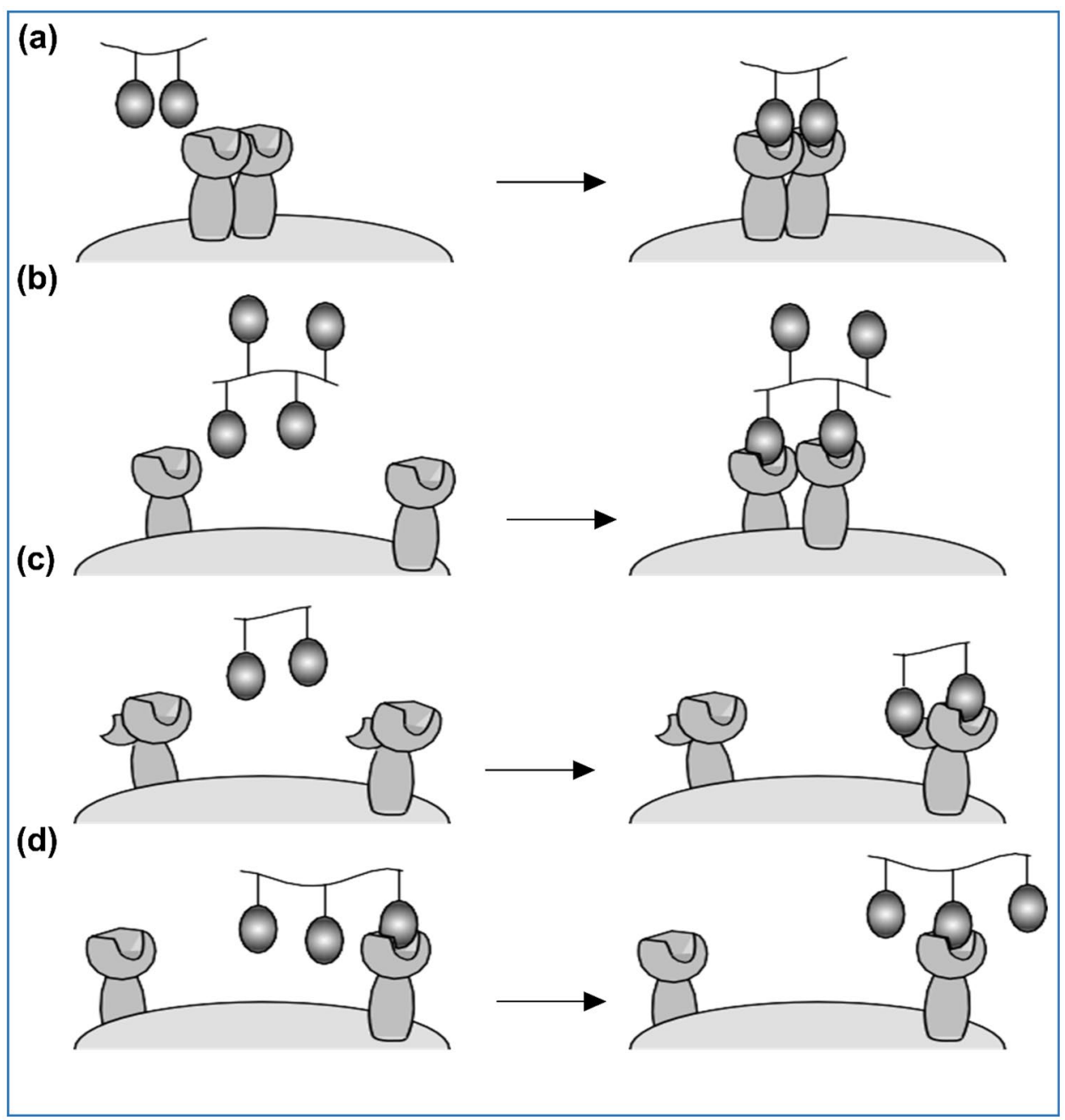




\section{Chelate effect}

Chelate effect or chelation accounts for the binding event in which multiple ligand molecules attach to multiple receptors at the same time, lowering ligand dissociation rate and boosting binding affinity, avidity, and stability of multimeric ligands [21]. Ideally, the binding enthalpy of the multivalent ligand is more favorable than that of its monovalent equivalent [69]. The translational entropy is compensated for the initial interaction between the receptor and multivalent ligand in this case; after that, binding interactions occur without further entropy compensation [24, 70]. The enthalpy of a system is an additive process according to thermodynamic principles, while the entropy of a multivalent system is not. Accordingly, the first binding interaction between the receptors and multivalent ligand causes the local concentration of neighboring ligands and receptors, which reduces the unfavorable entropic penalty paid for the binding interaction of ligands and receptors [69].

\section{Initiation of interaction by receptor clustering}

In this binding event, interaction between multivalent ligand (due to the near proximity of both ligand and receptor) and the target receptors results in the development of clusters within the receptor domain, further potentiating the multivalent interaction $[69,70]$. In other words, when ligands disassociate from receptors, multivalent ligands can rebind neighboring receptors rather effectively than monovalent ligands because of the clustering effect, which multimerically introduces binding sites. The successful clustering of these receptors also contributes to the signal transduction pathway being triggered [70].

\section{Steric stabilization of the binding event}

When multivalent ligands engage with their target binding sites, the physical dimensions of ligand molecules may impede the attachment of additional competing ligands to the target receptors. The steric stabilization is the method through which multivalent ligands and receptors interact [69]. In other words, the steric bulk of the multivalent ligand inhibits an opposing viral particle from attempting to connect with the target receptor [70].

\section{Subsite binding}

The process of multivalent interaction between ligands and target receptors that increases the chance of ligand-receptor binding is known as subsite binding. It is so because rotational and translational entropy was paid for the interaction between the first ligand and its binding site. Consequently, furthermore, ligand-binding occurs without loss of entropy $[21,24]$.

Monovalent ligands can only access and bind to the adjacent receptor site along with the primary binding site. Multivalent ligands, on the other hand, tend to obtain the requisite binding affinity, which is used for interaction with distant secondary binding sites. This form of interaction may be initiated by either a recognition epitope or a multivalent construct component [69].

\section{Statistical effect}

The statistical increase in ligand concentration caused by the multivalent arrangement of local ligands leads to the prominent binding affinity of these ligands. It potentiates the chances of ligand binding to their target sites provided by the high concentration of multivalent ligands surrounding the target receptor [21, 69]. These five binding events provide unique characteristics to the multivalent ligands. A brief overview of multivalency governing thermodynamic concepts continues to clarify the individual contributions of multivalent ligands to boost functional affinity towards the target receptor.

\section{Thermodynamic concepts behind the multivalent interaction}

\section{Role of cooperativity in the establishment of multivalent interactions}

Cooperativity typically occurs when the interaction of a ligand to a binding site influences additional ligands' binding. The term "cooperativity" is often used to describe biological phenomena, such as the binding of oxygen to hemoglobin to produce oxyhemoglobin, in order to evaluate the enthalpic alterations that occur (either positive or unfavorable process) in subsequent ligand-protein interactions [71]. Here, cooperativity positively contributes to the favorable free energies to bind each hemoglobin monomer with added oxygen. Cooperativity associated with a multivalent interaction can be quantified if the free energy associated with the multivalent interaction ( $\Delta E_{\text {multi }}$ ) is related to $M$ monovalent interaction, denoted as $M \Delta E_{\text {mono }}$, for $\mathrm{M}$ independent ligand and receptor binding event. The ratio between the two free energies indicates the cooperativity of the binding event, and the factor of cooperativity $(\beta)$ can be given as (Eq. 1)

$$
\beta=\text { Cooperativity }=\frac{\Delta E_{\text {multi }}}{M \Delta E_{\text {mono }}}
$$


Multivalent interactions depending on the magnitude of $\beta$ can be positive $(\beta>1)$, noncooperative $(\beta=1)$, or negative $(\beta<1)$. There are inherent variations between multivalent interactions and monovalent interactions, and techniques for determining cooperativity are inadequate for determining a multivalent binding event. Monovalent interactions in a multivalent cooperative scaffold are well established, but to assess polyvalent bonds' cooperation in multiple receptors is challenging to define [72].

To address this issue, Ercolani et al. [72] proved that cooperativity in multivalent interactions could only be assessed individually by inter and intramolecular binding events. An indication of the degree of cooperativity can be obtained by comparing the practical dissociation constant $\left(K_{\mathrm{d}(\text { practical })}\right)$ of a multivalent binding event concerning its theoretical dissociation constant $\left(K_{\mathrm{d}(\text { theory })}\right)$ as described in (Eq. 2).

$K_{\mathrm{d}(\text { theory })}=c K_{\mathrm{d}(\text { practical })} \times K_{\mathrm{d}(\text { intra })}^{(n-1)}$

where $c$ is the statistical factor for a binding event, $n$ is the multivalent construct's valency, and $K_{\mathrm{d}(\mathrm{intra})}$ is the hypothetical dissociation constant for the intramolecular binding event. As a result, if $K_{\mathrm{d}(\text { practical) }}$ is greater than $K_{\mathrm{d}(\text { theory)} \text {, }}$ then negative cooperativity is at work (theory). Similarly, if $K_{\mathrm{d}(\text { practical })}$ is smaller than $K_{\mathrm{d}(\text { theory) }}$, then positive cooperativity is the driving principle of multivalent interaction $[72,73]$.

Wolfenden et al. [74] showed cooperativity factor $(\beta)$ to estimate the intensity of the multivalent binding event in contrast with monovalent binding by exploiting the interaction of carbohydrate grafted dendrimers and lectin concanavalin $\mathrm{A}$. The extent of this binding interaction was estimated by assuming that the multivalent interaction's binding affinity is directly proportional to that of the monovalent binding event raised to the power $N$.

$K_{N}{ }^{\text {multi }}=\left(K^{\text {mono }}\right)^{\beta N}$

where $N$ is defined as the number of binding events; the researchers have demonstrated the enhancement of multivalent interaction by using the $\beta$ value of 1 throughout the multiple dendrimer generations. The study's results demonstrate that a multivalent binding event's binding affinity can be predictably influenced and may be tailored. These findings have offered a new regulation and predictability for synthetic multivalent constructs' design and development. Due to the challenges in applying the principle of cooperativity to explain multivalent binding events, multivalent ligand-receptor interaction has been measured to compare the functional affinity of a multivalent binding event with the comparable affinity of monovalent interaction.

\section{Role of avidity enhancement in multivalent binding events}

The molecular aspect of interactions between multiple ligands and receptors during a multivalent binding event is referred to as avidity. Multiple interactions result in increasing avidity of interactions (as shown in Fig. 3), resulting in the formation of a stable and robust bond between ligands and their target receptors [75].

The relative activity of the multivalent binding event in contrast with the monovalent binding can be expressed in terms of the ratio of avidity of multivalent interaction to monovalent affinity constant, described by Mammen and Whitesides [3] in Eq. (4).

$\alpha=K N_{\text {multi }} / K_{\text {mono }}$

$N$ denotes the theoretical number of binding events, and the relative strength of a multivalent binding event compared to the monovalent binding is represented by the avidity enhancement factor $(\alpha)$.

Based on this model's extension, Gargano et al. [76] utilized bivalent binding events between the ligand and receptors, assuming that $K_{1}, K_{2}$, and $K_{3}$ are equilibrium constants for the bivalent binding event. Derivation of every association or equilibrium constant is based on relative assumptions, the possible number of binding events, the potential for monovalent binding, and bound ligands' concentration.

$K_{b i}=K_{1} K_{2} K_{3}=2 \times 10^{-2}\left(K_{\text {mono }}\right)^{2}$

Equation (5) represents the overall association constant for a bivalent binding event. It provides an estimation of the interaction, obtained as a product of three association constants dependent on the relative binding event. And the enhancement of avidity can be represented as (Eq. 6).

$\alpha=\frac{K_{b i}}{K_{\text {mono }}}=2 \times 10^{-2}\left(K_{\text {mono }}\right)$

For the prediction of association constants for the multivalent binding event, the Eq. (5) can be rewritten as (Eq. 7)

$K_{\text {multi }}=F\left(s \times 10^{-2}\right)^{n-1}\left(K_{\text {mono }}\right)^{n}$

where $n$ is the associated valency of multivalent ligand, $F$ denotes the statistical factor associated with multivalent binding, and $s$ represents the distance between neighboring receptors. In a similar study, the association constant for the binding event between a pentavalent ligand and pentamer toxin was determined as represented by Eq. (8).

$K_{\text {penta }}=1\left(1 \times 10^{-2}\right)^{(5-1)}\left(K_{\text {mono }}\right)^{5}$ 


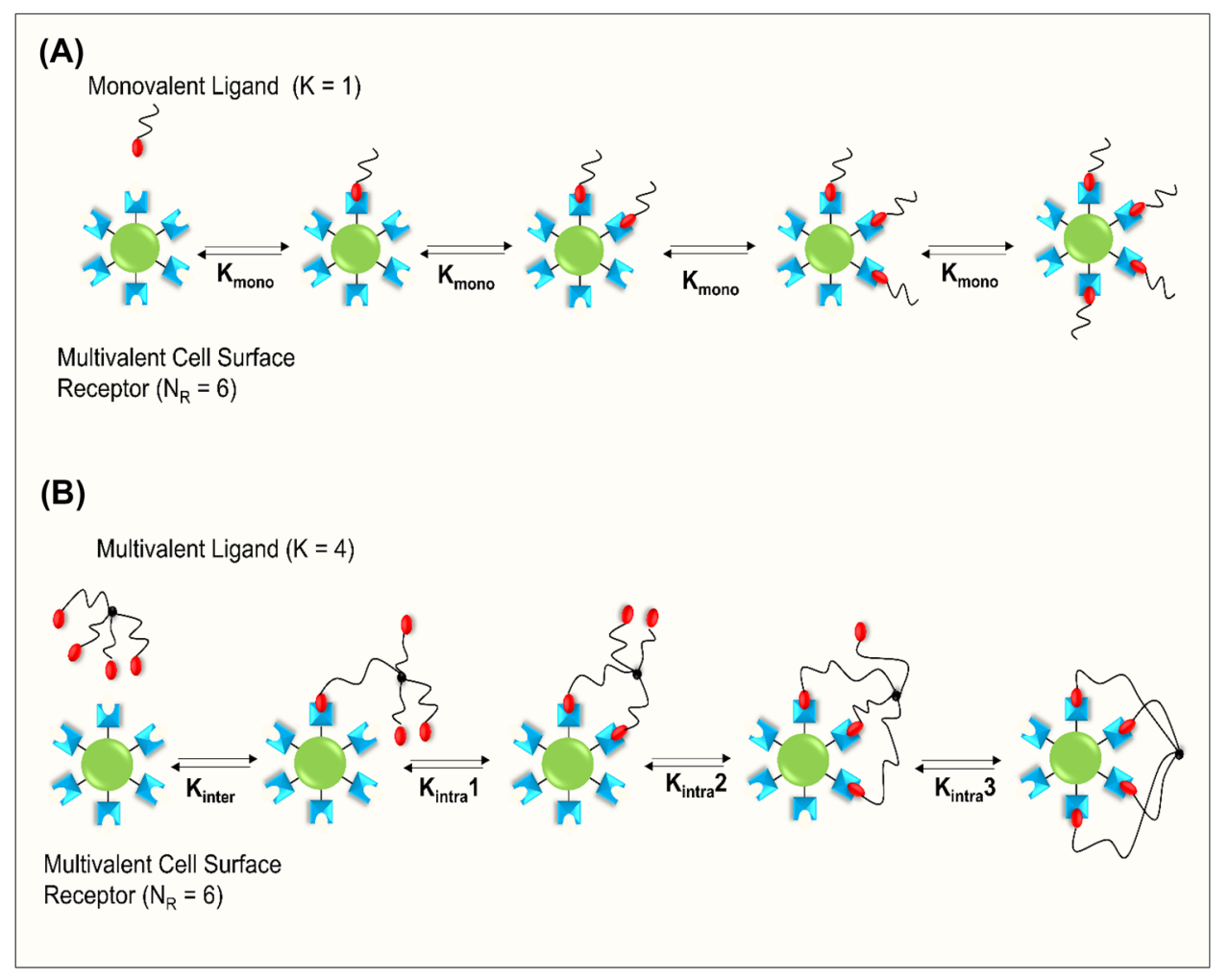

Fig. 3 Role of avidity enhancement. This scheme represents the multivalent receptors (light blue) randomly present on the cell surface and their interaction with targeting ligands (red), where multivalent ligands are assumed to be fully flexible. A The monovalent $(K=1)$ ligand binds to a multivalent receptor $\left(N_{\mathrm{R}}=6\right)$. There is no or little cooperativity associated with monovalent binding events; the individual binding constants $\left(K_{\text {mono }}\right)$ are all the same. Hence, the avidity

where taking the value of $F=1$ and $s=1$ and demonstrated $K_{\text {penta }}=1.2 \times 10^{7}$, Gargano et al. [76] have devised this model based on some assumptions, which are (a) all the interacting receptors are equivalent; (b) intramolecular binding leads to potentiation of the binding event; (c) the binding event takes place without cooperativity; and (d) binding event takes place without the deployment of a linker and spacer. Thus, the models for quantifying $K_{\text {penta }}$ and $K_{\text {multi }}$ demonstrate that a multivalent binding event's strength increases exponentially as the valency associated with the multivalent construct increases. Thus, expanding these concepts to even higher multivalent binding events may allow for the more effective design and development of multivalent constructs.

\section{Role of avidity entropy in the establishment of a significant multivalent interaction}

The avidity entropy represents a mathematical component that reflects the multivalent binding topologies (linear, radial, or circular). In other words, this component denotes the various aspects by which a particular binding event takes of the binding event is also constant. B A multivalent ligand $(K=4)$ binds to the same receptor as $\mathbf{A}$, with $N_{\mathrm{R}}=6$. The first step is intermolecular $\left(K_{\text {inter }}\right)$, not associated with cooperativity differs from the subsequent intramolecular $\left(K_{\text {intra }}\right)$ steps. In the presence of cooperativity $K_{\text {intra }} 3>K_{\text {intra }} 2>K_{\text {intra }} 1$, representing the progressive increase in intramolecular binding indicating avidity enhancement by the multivalent binding event

place. A thermodynamic model (Eq. (9)) was developed in a previous study to establish a quantitative approach for developing multivalent ligands that epitomize optimum avidity enhancements. This model uses three components to illustrate the enhancement of avidity associated with a multivalent binding event: (a) intrinsic free energy required for the intermolecular binding event $\left(\Delta G_{\text {inter }}^{\circ}\right)$, (b) free energy required for the intramolecular binding $\operatorname{event}\left(\Delta G_{\text {intra }}^{\circ}\right)$, and (c) a mathematical factor representing the probability of ligands association or dissociation per binding event $\left(\Omega_{i}\right)$, this component is referred to as avidity entropy, along with these components a weight coefficient $w_{i}$ accounts for the relative distribution of bound ligands at equilibrium [77].

$\Delta G_{\text {avidity }}^{0}=\Delta G_{\text {inter }}^{0}+\Delta G_{\text {intra }}^{0} \sum_{i=1}^{i_{\max }} \omega_{i}(i-1)+R T \sum_{i=1}^{i_{\max }} \omega_{i} \ln \frac{\omega_{i}}{\Omega_{i}}$

This thermodynamic interpretation considers the entropic energy required that is not hampered by the loss of conformational entropy that must be introduced as multivalent binding event proceeds. Researchers have 
developed a method to test this hypothesis by exploiting multivalent carbohydrate inhibitors and surface topologies of a multivalent construct to target Shiga-like toxin [77]. They concluded that binding events mediated by multivalent constructs having a radial arrangement of ligands demonstrate a higher affinity for the interaction manifested by intramolecular binding and avidity entropy. Thus, considering the results obtained from these studies demonstrates that avidity entropy plays a crucial role in establishing multivalent interactions.

\section{Role of effective molarity in showcasing the multivalent interaction}

In the "Modes of interaction between multivalent ligands and the target receptors" section of this review, we have mentioned that a multivalent binding event's statistical effect plays a significant role in ligand and receptor rebinding. The effective molarity or effective concentration is an important parameter to evaluate the statistical effect. Kinetics associated with ring closure intramolecular reactions are often estimated using effective molarity as a crucial parameter [78].

The intermolecular binding will favor a given multivalent interaction if the ligand's effective molarity is higher than the cell surface receptors in the solution. Previously, a study has been carried out to demonstrate that effective concentration $\left(C_{\text {eff }}\right)$ and inherent association constant $\left(K_{i}\right)$ are directly proportional to the association constant of the $\mathrm{n}$-valent binding event during multivalent binding events [79].

$K_{n}=b K_{i} C_{\text {eff }}^{n-1}$

In Eq. (10), $b$ is the factor for the statistical effect, which describes the number of binding events possible during an interaction. For binding events where multivalent interaction utilizes several noncooperative interactions, the effective molarity equals the effective concentration $\left(C_{\text {eff }}\right)$. This principle demonstrates a mode of the multivalent binding event, which is dependent on concentration. At low concentrations, intramolecular interactions are preferred, but intermolecular interactions are preferred at larger concentrations of multivalent ligands. Effective molarity of a multivalent construct grafted with $n$ ligands relies on the length and flexibility of the linker and is characterized by the ratio of inter-and intramolecular dissociation constants [79]. The authors extended this concept for use with higher-order multivalent constructs and then devised a model to estimate effective molarity based on the valency associated with the multivalent construct. Accordingly, effective molarity associated with a multivalent system comprising $\mathrm{n}$-valent intramolecular binding event having association constant $K_{n}$ can be represented as Eq. (11).
Effective Molarity $=\left(\frac{K_{n}}{b K_{i}}\right)^{\frac{1}{n-1}}$

It is evident in this context that the dissociation rate of the multivalent ligand ultimately depends on the bound ligand's concentration. The concentration of bound ligands also relies on the effective molarity for an intramolecular multivalent binding event [79]. It implies that nanoconstructs with high avidity and propensity to provide a reliable kinetic control can be tailored by using the concept of multivalency.

\section{Experimental methodologies for quantifying multivalency of a nanoconstruct}

A variety of analytical techniques have been used to investigate multivalent interactions in solutions and surfaces. The methods most extensively used to analyze such interactions are summarized in this section. The association and dissociation constants of ligands can be determined using spectrophotometric analysis [69], surface plasmon resonance (SPR) spectroscopy [70], and isothermal titration calorimetry [80], which can be used to quantify thermodynamic parameters associated with multivalent interactions. Atomic force microscopy (AFM) and ${ }^{1} \mathrm{H}$ NMR spectroscopy can be exploited to determine the binding constant between the multivalent ligand and the target receptor. The colloidal stability of multivalent nanoparticles can be determined by utilizing microscopy and dynamic light scattering techniques $[69,81]$. Surface plasmon resonance spectroscopy is widely used to quantify multivalent interactions associated with various biological systems. It allows for determining the binding constant associated with a multivalent binding event when a 1:1 binding state is established between a ligand and its target receptor [82].

Multivalent interactions can also be predicted by determining the $\mathrm{IC}_{50}$ (ligand concentration at which binding constant reduces by $50 \%$ of its initial value) by exploiting surface plasmon resonance spectroscopy [83]. Some of the established methodologies for quantifying multivalency associated with a system are mentioned in Table 1.

\section{Multivalent nanoconstructs for targeted drug delivery}

In the early 1900s, Paul Ehrlich introduced active targeting; since then, this concept has successfully targeted various diseases by modifying drug delivery systems to target specific cells. The multivalency concept has been used effectively to enhance on Paul Ehrlich's "magic bullet" concept. This section discusses the multivalent targeted drug delivery 
Table 1 List of established analytical techniques for quantifying multivalency

\begin{tabular}{|c|c|c|c|}
\hline Analytical technique & Quantifying parameters & Multivalent binding event & References \\
\hline Total internal reflection fluorescence & $\begin{array}{l}\text { Fluorescence intensity as a measure of the } \\
\text { amount of multivalent ligand }\end{array}$ & $\begin{array}{l}\text { 2D protein-protein interaction targeting a } \\
\text { model membrane }\end{array}$ & {$[84]$} \\
\hline NMR spectroscopy & $\begin{array}{l}\text { Diffusion coefficients from DOSY } \\
\text { measurements }\end{array}$ & $\begin{array}{l}\text { Hexameric resorcinarene and } \\
\text { pyrogallarene incorporated capsules }\end{array}$ & {$[85]$} \\
\hline $\begin{array}{l}\text { Surface plasmon resonance } \\
\text { spectroscopy }\end{array}$ & $\begin{array}{l}\text { Mass-dependent refractive index changes in } \\
\text { ligand-receptor interaction }\end{array}$ & Affinity screening of antibodies & {$[83]$} \\
\hline Quartz crystal microbalance & $\begin{array}{l}\text { Frequency change as a function of the } \\
\text { multivalent ligand concentration }\end{array}$ & $\begin{array}{l}\text { Multivalent interaction of lectins with a } \\
\text { crosslinked, surface-grafted } \\
\text { glycopolymer }\end{array}$ & {$[86]$} \\
\hline Transmission electron microscopy & $\begin{array}{l}\text { Number of multivalent ligands per } \\
\text { nanoparticles }\end{array}$ & $\begin{array}{l}\text { Multivalent carbohydrate-modified } \\
\text { quantum dots interact with lectins and } \\
\text { sperm protein }\end{array}$ & [69] \\
\hline Fluorescence microscopy & Fluorescence intensity as a function of time & $\begin{array}{l}\text { Binding of CdS quantum dots with variable } \\
\text { ligand multivalency of GABA receptors } \\
\text { on a cell membrane }\end{array}$ & [87] \\
\hline Dynamic light scattering & Hydrodynamic diameter as a function of time & $\begin{array}{l}\text { Aggregation of mono- and multivalently } \\
\text { thiol-stabilized gold nanoparticles in } \\
\text { solution }\end{array}$ & {$[81]$} \\
\hline $\begin{array}{l}\text { Surface plasmon resonance } \\
\text { spectroscopy }\end{array}$ & $\begin{array}{l}\text { Mass-dependent refractive index changes in } \\
\text { ligand-receptor interaction }\end{array}$ & $\begin{array}{l}\text { Affinity screening of antibodies } \\
\text { Multivalent association between selectins } \\
\text { and polyglycerol sulfates }\end{array}$ & {$[88]$} \\
\hline EPR spectroscopy & Dipolar interaction between spin probes & $\begin{array}{l}\text { Measurement of the distance between } \\
\text { multivalent spin probes }\end{array}$ & [89] \\
\hline Isothermal titration calorimetry & Heat as a function of the ligand: receptor ratio & Maltose and lactose grafted $\beta$-cyclodextrin & [90] \\
\hline Atomic force microscopy & $\begin{array}{l}\text { Force as a function of the intermolecular } \\
\text { distance }\end{array}$ & $\begin{array}{l}\text { Molecular interaction between } \\
\text { bacteriophages and lipopolysaccharide } \\
\text { bilayers }\end{array}$ & [91] \\
\hline $\begin{array}{l}\text { High-performance liquid } \\
\text { chromatography }\end{array}$ & $\begin{array}{l}\text { The difference in retention as a function of } \\
\text { polarity and molecular size }\end{array}$ & $\begin{array}{l}\text { Multivalent interaction between } \\
\text { vancomycin and d-Ala-d-Ala trimer }\end{array}$ & [92] \\
\hline Circular dichroism spectroscopy & $\begin{array}{l}\text { Circular dichroism intensity as a function of } \\
\text { the ligand and receptor concentration }\end{array}$ & $\begin{array}{l}\text { Hybridization of oligothymine templates } \\
\text { with oligomeric adenine and naphthalene } \\
\text { diaminotriazine as ligands }\end{array}$ & [93] \\
\hline
\end{tabular}

nanoconstructs that have been rationally engineered for targeted drug delivery.

\section{Dendrimers}

Dendrimers are 3-dimensional-branched structures that are synthesized generation after generation. Dendrimers may play drug carrier roles in nanometer dimensions and efficiently exhibit targeting ligands on their surface due to their nanoscale and multifunctional structure [94, 95]. Dendrimers provide an attractive synthetically targeted multivalent system for therapy in cancers and cardiovascular diseases $[96,97]$. The multivalent dendrimers can be formed by (i) assembly of functionalized components and the formation of the polymer backbone or (ii) reaction after the polymer scaffold has been assembled. Multivalent dendrimers may be formed by following two different processes: first procedure (i) involves the aggregation of functional components along with the development of the polymer backbone, and the second procedure (ii) involves the attachment of the functional components after the synthesis of the polymer backbone, mediated by the help of a reaction. Procedure (i) demonstrated the convergent synthesis of dendrimer [98-101] (Fig. 4a) and polymerization of functionalized oligomer (Fig. 4b) units with pre-attached drugs or targeting moieties. Procedure (ii) demonstrates the use of divergent synthesis of dendrimer [102-105] (Fig. 4c) and the synthesis of functionalized hyperbranched polymer (Fig. 4d). Convergent approaches offer numerous advantages in terms of regulating conjugation efficiency and scaffold diversity [106-108]. Dendrimer generated in this manner seems to have fewer incomplete branches, and synthesis procedures can typically be carried out with stoichiometric quantities of reagents. However, the divergent synthesis demands a significant excess of reagents. If the convergent synthesis mechanism arises from the functional ligand, the diversity of the conjugated functional group in the finished product is prone to be associated with scaffolding defects, and these defects are consistently seen on dendrimers of lower generation. The main drawback of this approach is the efficient 


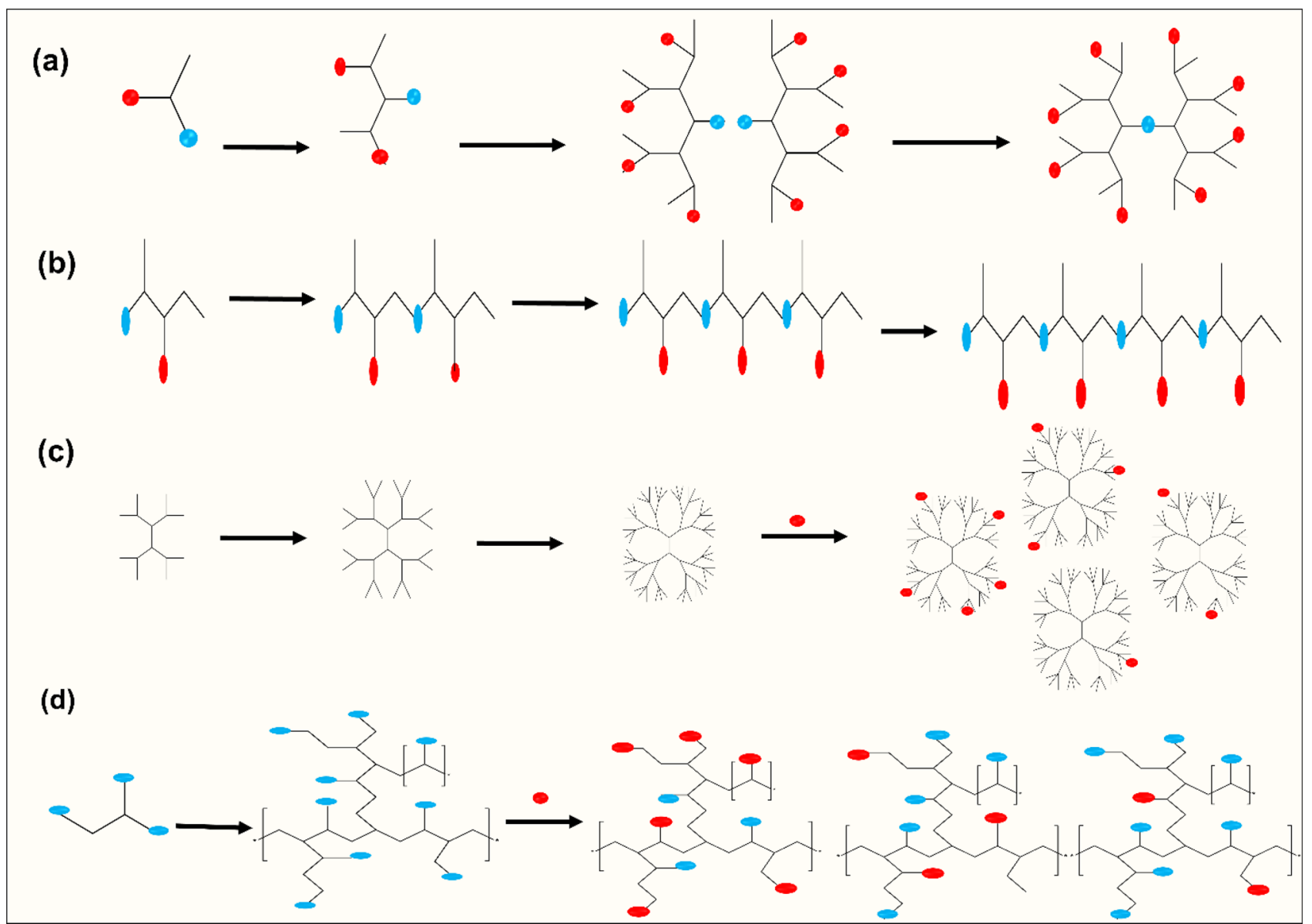

Fig. 4 Schematic illustration of procedures to be followed for the synthesis of multivalent dendrimer conjugates. (a) Convergent dendrimer synthesis allows for precise regulation but only allows for minimal size and valency. (b) Precise variation of regiochemistry can be achieved by bottom-up synthesis. However, this approach is confined

accomplishment of the primary coupling step; as dendrimer generation grows, the requirement of functional groups becomes multiple of the number of arms present (Fig. 4a). The complexity in executing the core coupling step as a generation grows results in a cumulative polymer dimension and molecular weight constraint. Methodologies to fix this issue include a blended approach that incorporates divergent and converging strategies [109]. Another prospective convergent strategy will be the bottom-up oligomer synthesis to have several copies of the ligand or attachment sites inside the backbone of the oligomer (Fig. 4b), for instance, a sequence-specific attachment of a click ligand to the peptide backbone [110]. This method is also constrained by the expense of synthesis and is restricted to only short oligomers. Ligands can be conjugated with preformed polymers to produce the desired ligands-to-scaffold ratio, usually, with the attachment of a single or sequential ligand to the polymer backbone (Fig. 4c, d); this type of arrangement permits only to oligomers. (c) The divergent mechanism of dendrimer synthesis and (d) linear comb or hyperbranched polymers allow for the synthesis of larger polymers but are associated with random valence statistics

more extensive polymer scaffold synthesis (i.e., divergent dendrimer synthesis and traditional polymerization techniques) [108].

Polymer scaffold provides several sites which are available for chemical modification, e.g., poly-(amidoamine) (PAMAM) dendrimers have (hypothetically) 4-4000 primary amines, based on generation (G1-G11), available for peptide coupling $[111,112]$. Such scaffolds have the advantage of providing multiple potential conjugation sites and high molecular weight, which makes many hydrophobic ligands soluble. However, the conjugation leads to a statistical distribution of ligand to scaffold ratios.

Chabre et al. [14] were among the first few researchers to describe multivalent glycodendrimers. Their dimensions currently range from traditional small frameworks to particles with more than $10 \mathrm{~nm}$ size. Dendrimers have a much more organized shape than linear polymers because dendrimer growth may be controlled before the reaction 
commences [15, 113]. Small dendrimer molecules have discrete structures but may be less efficient for the display of multivalent effects. Large-sized dendrimers show a low polydispersity index than other polymeric structures but are not as homogeneous as smaller dendrimers. According to Chabre et al. [14], optimizing ligand density on polyamidoamine dendrimers with a 50\% carbohydrate ligand resulted in the most potent activity towards concanavalin A. Moreover, the hemagglutination study revealed that the binding interaction of monovalent glycodendrimers of smaller sizes with concanavalin A increased by $2-3$ magnitude orders when compared with bivalent glycodendrimer system with larger dendrimers. Furthermore, the statistical effects of the multivalent binding event associated with glycodendrimer have been shown to increase the overall glycodendrimer activity [16].

In another study, Wang et al. [114] synthesized mannose grafted multivalent dendrimers to develop the human immunodeficiency virus (HIV) vaccine. The proposed target of the dendrimer is gp120 (a glycoprotein overexpressed by HIV). Mannose-grafted dendrimers have shown an increased affinity towards HIV antibody (2G12); the glycoconjugates have demonstrated 104 times more prominent $\mathrm{IC}_{50}$ values than the monovalent construct. These dendrimers are also tested for their binding affinity by flow cytometry analysis, indicating that multivalent dendrimers can bind to target receptors with higher affinity than monovalent counterparts [114]. Dendrimers are a promising multivalent carrier system for biomedical and therapeutic applications; their flexibility allows them to efficiently lower the entropy cost associated with the multivalent binding event. Moreover, the size of the scaffold can be easily altered by altering the generations of the dendrimer. Overall, dendrimers are excellent synthetic carrier systems for the multivalent display of molecular diagnostics and therapeutic agents.

\section{Nanoparticles}

Multivalent ligand-conjugated nanoparticles have been designed to demonstrate the hybrid action of the encapsulated drug and to coordinate the system with the target cell. Nanoparticles have various advantages, including the capacity to carry a high drug payload and the ability to shield drugs. Targeted delivery of cisplatin-loaded PLGA-PEG nanoparticles grafted with multivalent pentapeptide to $\alpha_{v} \beta_{3}$ integrin receptor has demonstrated enhanced internalization in prostate and breast cancer cells [115]. The improvement in the affinity of multivalent RGD peptides grafted on nanoparticles to $\alpha_{v} \beta_{3}$ integrin has been documented previously. RGD peptides' binding to nanoparticles resulted in prolonged circulation time from 13 to $180 \mathrm{~min}$ due to affinity enhancement by multivalent interaction.
Moreover, the multivalent RGD peptide grafted nanoparticles with an $\mathrm{IC}_{50}$ value of $1 \mathrm{nM}$ had a multivalent enhancement factor of 38 [116]. In a study, vancomycin-loaded nanoparticles have been prepared by grafting a specific multivalent peptide to target Staphylococcus aureus effectively. Hussain et al. [117] demonstrated that multivalent vancomycin-loaded nanoparticles effectively treat Staphylococcus aureus-infected tissues and produce successful results with ten times lesser vancomycin concentration than the treatment using free vancomycin. It is an important finding as vancomycin is associated with producing severe toxicity events. Kiziltepe et al. [118] prepared doxorubicinloaded micellar nanoparticles targeting very late antigen-4 (VLA-4) receptors by grafting multivalent VLA-4 antagonistic peptides. The results demonstrated that multivalent VLA-4 antagonistic peptide-conjugated nanoparticles are associated with lower systemic toxicity, potentially inhibited tumor growth, and accumulated more drugs at the target site (in vitro) than unconjugated nanoparticles. The binding of targeted multivalent nanoparticles to VLA-4 was achieved by receptor-mediated endocytosis with an optimal valency of 20 peptides per nanoparticle, effectively indicating the role of ligand valency in designing effective multivalent nanoconstructs for targeted drug delivery.

A multivalent magnetic nanoparticulate system that utilizes magnetic resonance imaging (MRI) for tumor treatment was designed in a recent research. Zhang et al. [119] used a dual-acting agent raltitrexed as a ligand to target the folate receptor. Different valencies of raltitrexed-modified multivalent polyethyleneimine ligand cluster $\mathrm{PR} n(n=2,4$, and 8) were conjugated on magnetic nanoparticles to form multivalent magnetic nanoparticles with variations in their valency. The in vitro studies demonstrated that PR4 was the most effective valency in treating high folate receptor overexpressing KB cells with a decentralized receptor distribution. The PR2 was negative in statistical rebinding, and PR8 could induce steric hindrance in the limited binding area, inferring that ligand valency with a relatively superior therapeutic effect was associated with the folate receptor overexpression level. Along with these findings, they have also demonstrated the utility of multivalent magnetic nanoparticles to serve as an MRI contrast agent for the diagnosis of liver carcinoma. Hence, this study effectively demonstrates the utility of multivalent nanoparticles both as a therapeutic and a theranostic system for targeting different types of tumors. Abstiens et al. [120] recently evaluated RGD peptides with varying ligand densities as well as different PEG spacer lengths surface grafted onto core-shell nanoparticles to determine the critical role played by both ligand density and linker length in the successful establishment of a multivalent ligand-receptor binding event. Authors have reported that nanoparticles with $100 \%$ RGD peptide surface grafting and short PEG linkers exhibited a higher tendency to form 
clusters, allowing for a cooperative ligand-receptor binding event on the cell surface. Contrastingly, longer PEG linkers with higher flexibility increased the chances of ligand entanglement, reducing the magnitude of the ligand-receptor binding event. As a result, as described in the preceding sections, a study by Abstiens et al. [120] strongly suggested in a similar way that in order to optimally apply the principle of multivalency, the design and development of nanoparticles in terms of ligand density and linker length must be adequately optimized. In a similar study, Maslanka Figueroa et al. [121] demonstrated that by adjusting the polymer composition on the nanoparticles, one might modify ligand flexibility and, as a result, endocytosis of nanoparticles. The authors demonstrated that nanoparticle surface containing angiotensin-II (ligand) conjugated to a long polyethylene glycol chain separated by a ligand-free short polyethylene glycol chain internalizes at a faster rate in angiotensin-II receptor type-1 positive cells. However, when the flexibility of ligand is hindered by dense surface grafting of nanoparticles only with ligand conjugated long polyethylene glycol chain, the resulting cellular internalization was reduced by a magnitude of $50 \%$. These results demonstrate that developing multivalently-binding nanoparticles is a complex procedure that involves striking an equilibrium between various particle properties. Among these, the flexibility of ligands, which can be enhanced by changing the polymer composition and the number of targeting moieties surface grafted onto the nanoparticles, must be considered to establish optimum cellular interactions. Furthermore, Karimi et al. [122] also discussed the fabrication of multivalent nanomaterials in-depth with explanations and details.

\section{Antibodies}

Antibodies such as anti-CD20 and CD22 antibodies can target various cytotoxic compounds such as drugs, radionuclides, or toxins for therapy [123-125]. The multivalent antibodies showed different properties than monovalent counterparts, including prolonged half-life and enhanced selectivity to targets $[126,127]$. Due to the specificity of antibodies to tumor-associated antigens on malignancies, antibodies have been used as targeting ligands or as tumortargeting delivery vehicles, also known as immunoconjugates $[125,128]$. Previous literature discussed the "dock and lock" mechanism for creating multivalent proteins. This technique was designed to prepare bispecific antibodies that link divalently to a tumor-specific antigen and monovalently to a radiolabelled hapten peptide for pre-targeted diagnostics and therapy (Fig. 5A), but it can also generate a variety of other biologic agents of therapeutic significance [129].

Several multivalent antibody constructs have been developed by exploiting anti-CD20 and CD22 antibodies, and the results suggest that multivalent antibodies have somewhat different characteristics than their monospecific counterparts. Authors have reported that the technique's utility is restricted to multivalent antibodies, but other potential multivalent targeting agents (interferon $\alpha$ ) can also be created (Fig. 5B and C). These scaffolds are reported to provide a dual function: first, to sustain the system for a prolonged period in the systemic circulation, and second, selective targeting [129].

The multivalent antibody conjugates have been studied to enhance ligand affinity, alter the pharmacokinetic profile, and improve stability [130]. Multivalent single-chain variable fragments ( $\mathrm{scFv}$ ) have been shown to increase the binding affinity to tumor-associated antigens and improve in vitro and in vivo stabilities of the construct [131]. Delalat et al. [132] utilized the exposure of multivalent antibodies to bioengineered biosilica diatoms to invade tumor cells. Diatoms are microorganisms that comprise silica-based cell walls, and their use could be favored over synthetic silica due to the expense and hazardous materials used in silica manufacture. Diatoms have been biologically modified to express GB1 (that attaches IgG) on their biosilica layer. The GB1 allowed the conjugation of cell-specific antibodies. The drugs were first encapsulated into liposomes to load drugs onto a bioengineered diatom. Since the liposomes used were positively charged, and biosilica was negatively charged, multivalent electrostatic attraction enabled the drug-filled liposomes to bind to the diatom [132]. The results revealed that the drug-loaded, IgG-presenting diatom silica could be used to reduce the tumor volume significantly. At the same time, non-target liposomes with the entrapped drug showed little or no effect. Recently, in the context of the current pandemic situation caused by a novel corona virus (SARS-CoV-2), Rujas et al. have demonstrated that the concept of multivalency can transform antibodies against SARS-CoV-2 into ultrapotent neutralizers by combining antibodies of three different specificities and fragment crystallizable domain on a single multivalent entity (Multabody) provided the capacity to overcome the viral sequence diversity, as well as exceptional efficacy along with IgG like bioavailability. Authors have reported that "Multabody" platform harnesses binding avidity and multispecificity to deliver 10,000-fold more potent broad-spectrum neutralizers against SARS-CoV-2 when compared to IgG counterparts, making them promising therapeutic options for targeting SARS-CoV-2 [133].

\section{Self-assembling nanoconstructs for drug delivery}

This segment outlines examples of self-assembling nanoconstructs, including virus capsids and synthetic vesicles, which either assemble into stealthy structures or have triggered activity. A study has documented that viral capsids functionalized with carbohydrate ligands have outstanding lectin binding properties [134]. Viral capsids have also been identified as 
Fig. 5 A Schematic representation of pretargeting a tumor vasculature using a multivalent bispecific antibody and a radiolabelled hapten (di-HSG) peptide. The bispecific antibody is intravenously administered. After its localization into a tumor, a hapten (di-HSG) peptide is being administered. A marked enhancement in avidity is reported under the crosslinks formed between adjacent bispecific antibodies. B Schematic illustration of multivalent antibodies or multivalent antibody-interferon scaffolds prepared by exploiting dock and lock technique. The heavy chain of an anti-CD22 Immunoglobulin is modified by incorporating AD2 peptide sequence, producing an immunoglobulin with two AD2 sequences. A homodimer, Fab-DDD2 of anti-CD20 antibody will bind and lock on the immunoglobulin AD2 structure, leading to the formation of the multivalent scaffold with four anti-CD20 Fab binding moieties. C The same immunoglobulin AD2 scaffold is linked to interferon- $\alpha$. This modification leads to the formation of a multivalent immunoglobulin scaffold competent in targeting four interferon- $\alpha$

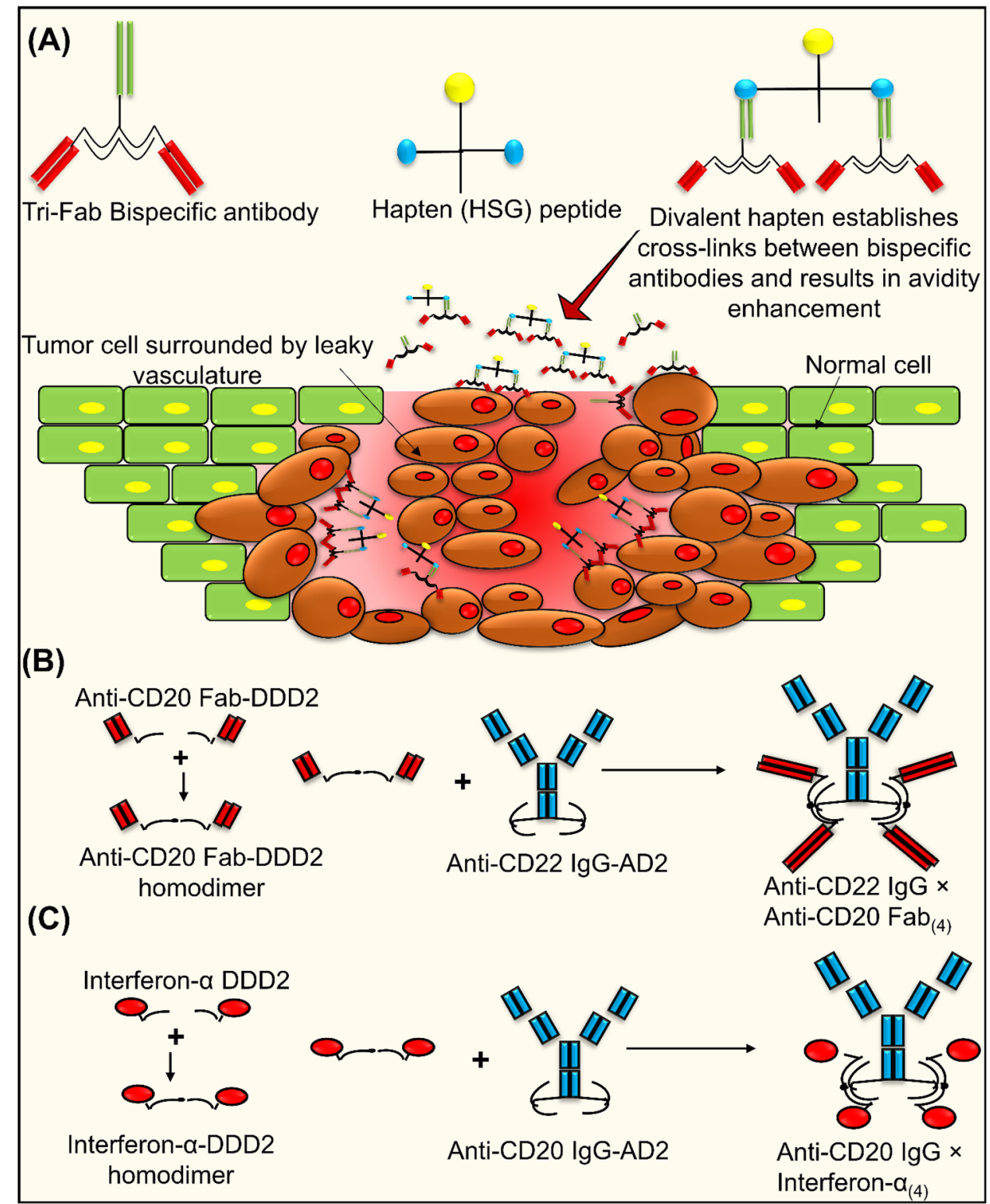

contrast agents in MRI; capsids may contain several gadolinium centers, providing a multivalent epitope for targeting [135]. Previously, Kim et al. [136] previously reported the formation of cylindrical and spherical vesicles and micelles of varying sizes. When carbohydrates were exposed, the estimated binding affinity of the protein-carbohydrate binding event with these nanoparticles improved significantly. Simnick et al. [137] demonstrated self-assembly by induced heat stimuli using an elastin-like polypeptide that forms micelles at critical micelle temperature.

These induced multivalent nanoconstructs displaying RGD peptides recognize and bind $\alpha_{v} \beta_{3}$ integrins and have been shown to exhibit "dynamic amplification of the receptor binding affinity in response to an external stimulus." Stimulus-promoted self-assembly and careful dismantling can emulate physiological multivalency and provide nanoconstructs with suitable circulation time. It may be beneficial in therapeutic systems such as the delivery of drugs.

\section{Sperm cell-mediated targeted therapy}

Instances of multivalent binding events are often found naturally and can be exploited for drug delivery. Xu et al. [138] used sperm cells as a drug delivery vehicle for targeted drug delivery; among some of the significant advantages of sperm as a carrier is its ability to fuse or attach with cells, facilitating multivalent interactions between several sperm proteins and target cellular membranes. This fusion capability was expected to increase drug transport to tumors. Researchers have shown that delivering drugs incorporated in sperm to 
HeLa cell spheroids is more effective than spawning HeLa cells in a doxorubicin solution. Another feature of the sperm is its ability to drive itself, and so, the researchers produced an iron-coated "tetrapod" that enclosed the sperm's head so that its travel could be guided through a magnetic field. They demonstrated the capability to control sperm movement through microfluidic channels to deliver doxorubicin hydrochloride to the HeLa cell spheroid. HeLa cell spheroid's size decreased by $40 \%$ in $8 \mathrm{~h}$ after drug delivery.

\section{Nanoworm-mediated targeted drug delivery}

Another fascinating system that uses a multivalent peptide interface for targeted cancer therapy is the "nanoworm" [139]. Nanoworms are composed of iron oxide nanoparticles that always form an elongated worm-like structure and multivalently exhibit covalently linked recombinant peptides. Such recombinant peptides have both targeting and an apoptotic domain. The targeting domain binds explicitly to $\mathrm{p} 32$, a protein located in vascular endothelium that shapes tumor vasculature. The targeting domain also comprises a sequence that enables the nanoworm to invade the perivascular tissues of the tumor. Interestingly, the targeting peptide used in the finished nanoworm system exhibited hardly any ability to target p32 as a monomer but was successful when multivalently grafted on the nanoworm. Researchers credit the principle of multivalency for the potentiation of peptide activity on nanoworm. The later domain of recombinant peptide, the apoptotic domain, can effectively damage mitochondrial membranes and thus act as a drug within the nanoworm (Fig. 6). Agemy et al. [140] stated that perhaps the multivalent presentation of the apoptotic domain [KLAKKLAK] 2 on nanoworm results in increased potency relative to the monomeric [KLAKKLAK]2. The recombinant nanoworm was screened in glioblastoma-bearing mice, which effectively decreased tumor volume. Other than the iron oxide nanoparticles nanoworm, Aluri et al. [141] developed hybrid single-chain variable regions of anti-CD20 and elastin-like polypeptide polymer nanoworm and studied the efficacy of nanoworm on two cell lines of B cell lymphomas compared to Rituximab, which induces cell death via binding and clustering of CD20 receptor. The nanoworm showed

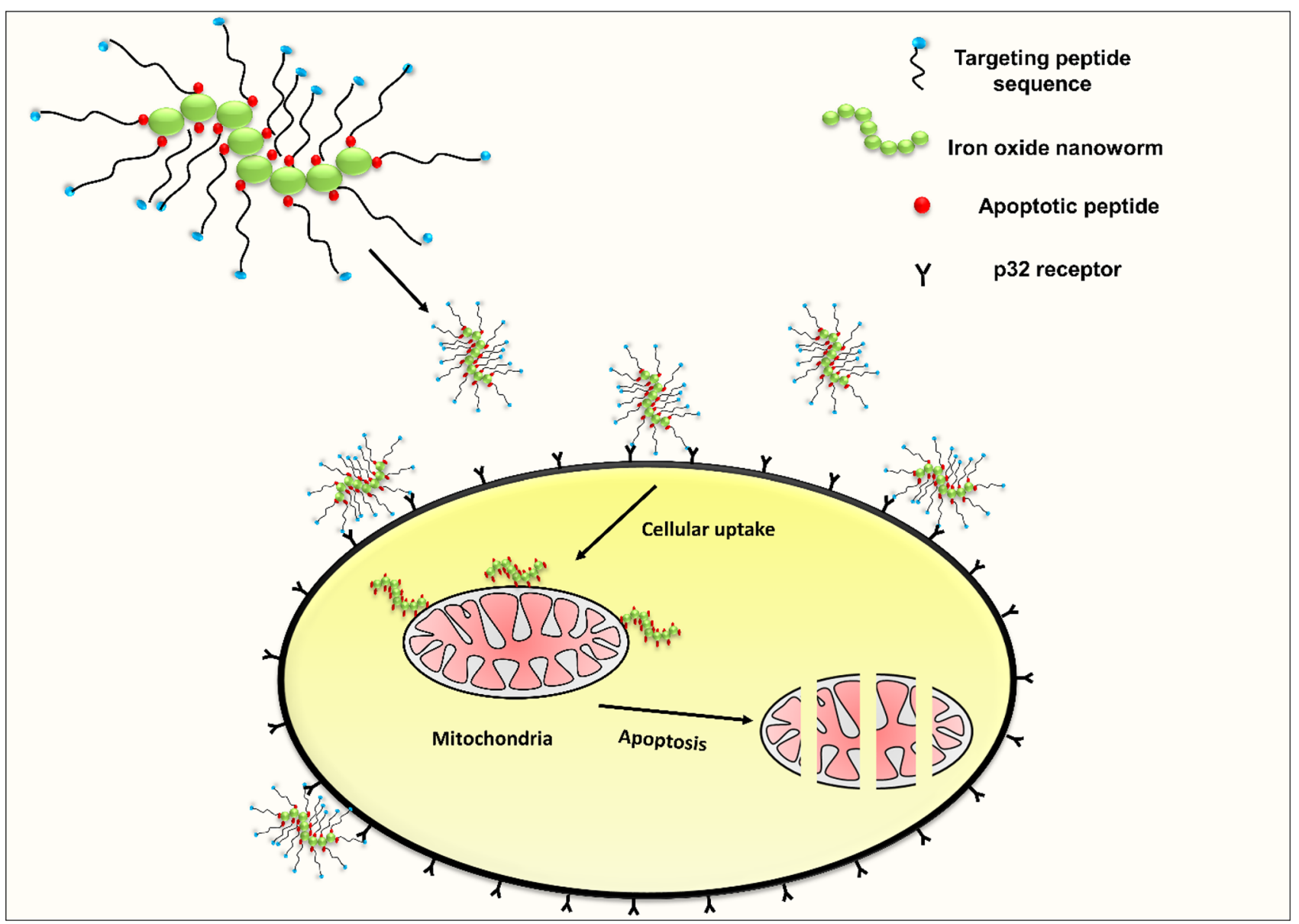

Fig. 6 Schematic representation of the nanoworm and its mechanism of action 
better efficacy than Rituximab alone causes apoptosis in a concentration-dependent manner. Similarly, Lee et al. [142] designed nanoworm by single-chain variable regions and elastin-like polypeptide to target the B cell and T cell receptors for non-Hodgkin lymphoma.

\section{Proteins as a biological nanomaterial for cancer targeting}

Proteins are of great importance as far as multivalency is concerned. Since their nanoscale dimension enables them to encompass vast surface area allowing the chelate effect to take place, along with this, the well-defined system of proteins allows for specific functionalization. Moreover, proteins are associated with intrinsic therapeutic potential. Luo et al. [143] used a tetrameric red fluorescent protein as a scaffold and an imaging agent onto which peptides for cancer targeting were multivalently conjugated. The $\mathrm{N}-\mathrm{C}$ terminals of each tetrameric red fluorescent protein unit have been precisely conjugated with targeting peptides to produce conjugates of exactly eight targeting peptides per tetrameric red fluorescent protein. The conjugation of the peptides to the tetrameric red fluorescent protein drastically improved the endocytosis of the probe. The magnitude of fluorescence of the tetrameric red fluorescent protein, on the other hand, was shown to decrease. To resolve this problem, Kostiainen et al. [144] used dendrons of various generations to build DNA binding patches of variable valency on two distinct protein scaffolds, bovine serum albumin and genetically modified class II hydrophobin. This study utilizes the advantage of a solitary cysteine residue available on every protein mediating the thiol reaction with dendrons. The use of dendrons with three or nine primary amine surfaces allows for accurate valency regulation of the final conjugate. Even though the bovine serum albumin scaffold yield was only $50 \%$, attributable to cysteine oxidation, the processed products represented a solitary dendron for every protein. An ethidium bromide displacement analysis then assessed the DNA coupling of the conjugates. The conjugates displayed binding interaction with DNA with varying attributes, while unmodified proteins did not display the binding interaction. The smaller genetically modified class II hydrophobin conjugates had a comparatively higher affinity than larger bovine serum albumin conjugates, correlated to variations in the dendron-to-protein dimension.

\section{Multivalent aptamers as "nanocentipede" and "nanorobots" for targeted drug delivery}

Aptamers are another class of molecules that are frequently introduced multivalently for targeted drug delivery. Li et al. [145] reported the role of multivalent application of aptamers for targeted cancer therapy by developing a system called "nanocentipede." The nanocentipede was assembled and functionalized using the hybridization chain reaction of two biotin-conjugated nucleic acid monomers; streptavidinconjugated ZY1 aptamers targeting SMMC-7221 cells were linked (Fig. 7A). Furthermore, characterization of the nanocentipedes revealed that perhaps the avidity of the nanocentipede for target cells improved with a multivalent display of aptamer. In addition, an improvement in endocytosis could be seen for multivalent nanocentipedes relative to monovalent nanocentipedes (Fig. 7B). The researchers then displayed the capability to load doxorubicin further into the nanocentipede trunk so that the system could be used for targeted drug delivery (Fig. 7C) [145].

$\mathrm{Li}$ et al. [146] employed multivalent aptamers to infiltrate tumors, and when bound to their target, such aptamers act as a catalyst for drug presentation. They named the formulation "Nucleic acid nanorobot." The nanorobot consists of a nucleic acid origami substrate conjugated to thrombin, a proteolytic enzyme that causes blood clotting. This nucleic acid substrate could be turned into a nanotube and secured with multivalent nucleic acid surface fasteners so that the thrombin was trapped in the nanotube. The nucleic acid fasteners are composed of two DNA strands, one strand, including that of the aptamer AS1411, about which the second strand was partly complementary. The aptamer AS1411 specifically binds with nucleolin, which is mainly upregulated on several tumors' cell surfaces. As a result, they are binding to nucleolin causes the opening of the nanotube to release thrombin effectively. Additional AS1411 aptamers for enhanced multivalent targeting have been applied to the ends of the nanorobot. The researchers utilized several mice tumor models to examine the efficacy of DNA nanorobots in vivo, including one with a skin cancer mouse model in which mice treated with nanorobots substantially higher survival those treated with saline, natural thrombin, or blank nanorobots.

\section{Effective internalization of multivalent drug delivery nanoconstructs}

With evolving varieties of nanoconstructs designated for physiological applications [147], substantial endeavors have been undertaken to understand these nanoconstructs' destiny in vitro and in vivo. The underlying mechanisms for the internalization of these nanoconstructs into cells are of specific importance for understanding their molecular mechanisms. Plenty of researchers have used specific chemical interceptors to study intracellular trafficking of these nanoconstructs, which is extremely important for identifying and quantifying target receptors and providing valuable information for the design and development of multivalent nanoconstructs [148]. Dalal et al. [149, 150] explored the role of multivalency in subcellular trafficking of $35-50 \mathrm{~nm}$ nanoparticles with a valence range of 10-40 in nanoparticle-based imaging 
Fig. 7 Multivalent nanocentipede. A The nanocentipede is assembled by exploiting the hybridization chain reaction of two biotinylated DNA sequences ( $\mathrm{H} 1$ and $\mathrm{H} 2)$ to which ZY1 aptamers (represented by legs of nanocentipede are multivalently arranged) conjugated with streptavidin targeting SMMC-7721 cells are attached. B Confocal microscopy assessed the cellular internalization of monovalent (Mono-Zy1-Nces) and multivalent (Zy1-Nces) nanocentipedes in SMMC-7721 cells. C Drugloaded nanocentipede multivalently interacts with the target cellular receptors followed by increased cellular uptake by multivalency resulting in enhanced cytotoxicity (reused from Li et al. [145] permission with American Chemical Society, Copyright 2016)
(A)

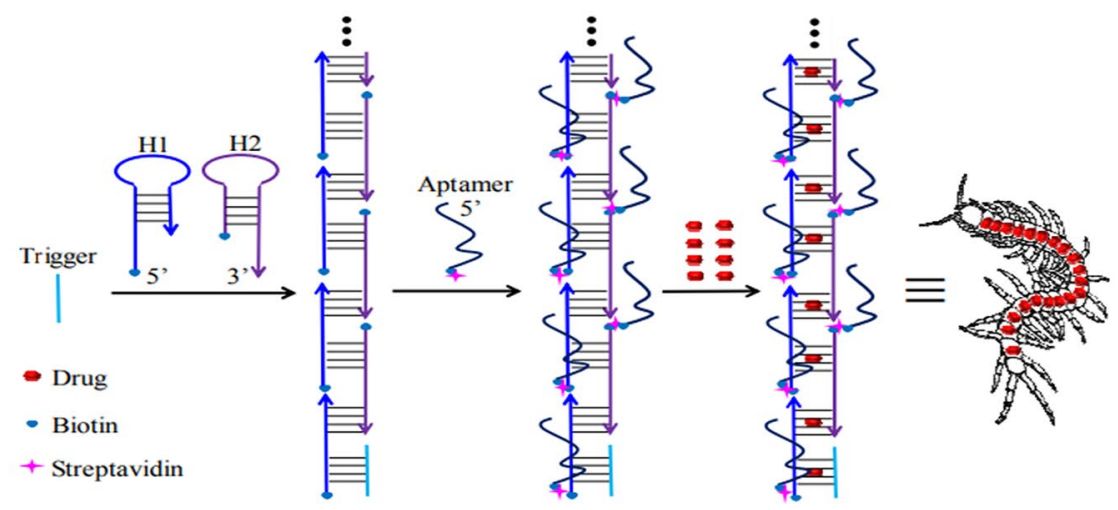

(B)
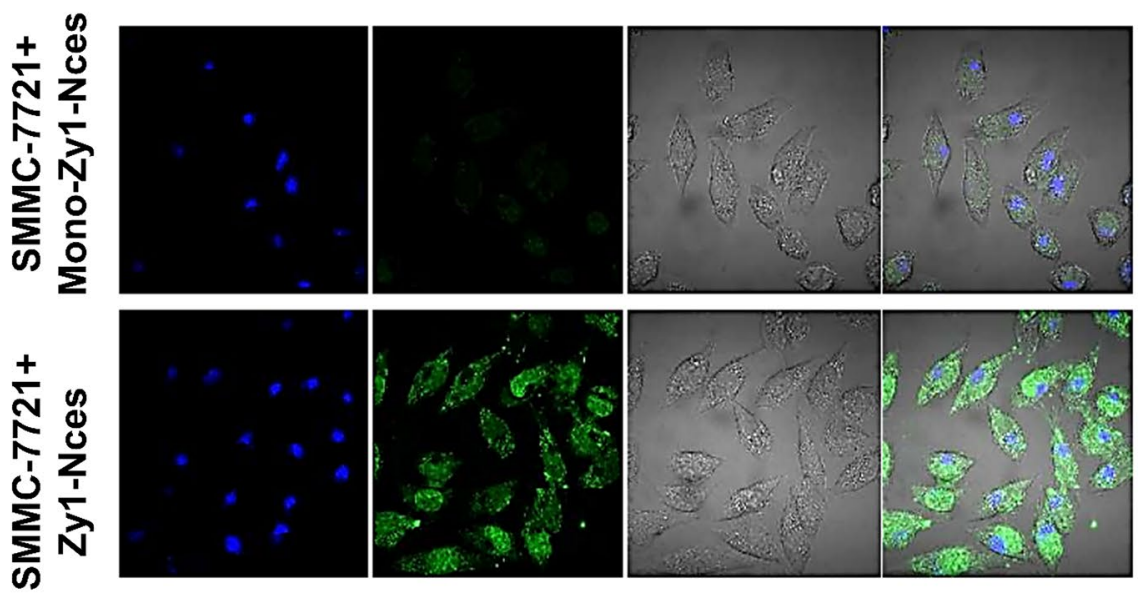

Lyso Tracker Blue

\section{Sybr Green Bright Field}

Merged

(C)

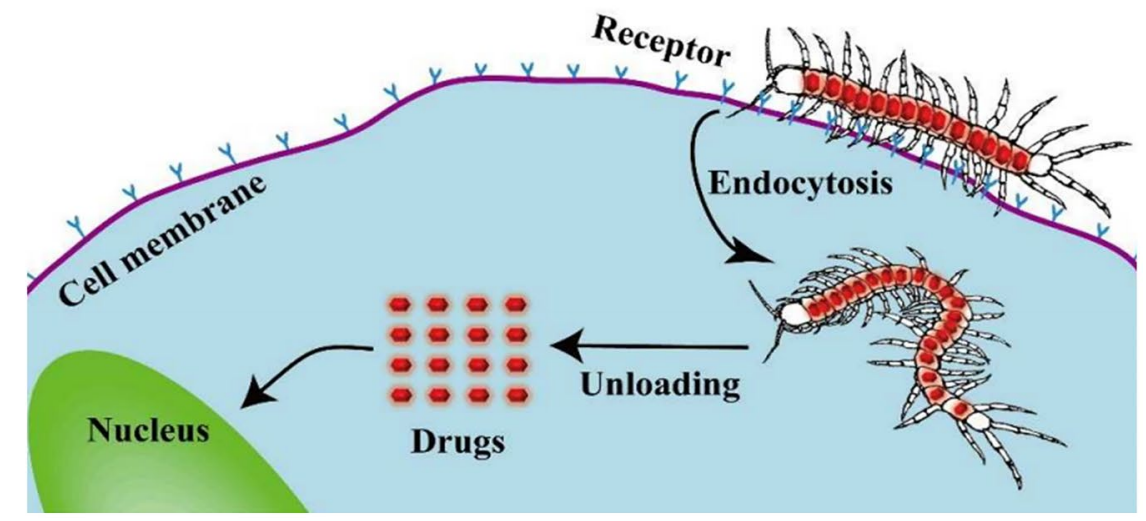

agents' construction. They reported that particles with relatively high multivalency (40 ligands per particle) had entered the cell through clathrin-mediated endocytosis. The lysosomal transportation of these nanoprobes inhibited their subcellular targeting. At the same time, the particles with relatively low multivalency (10-20 ligands) that entered the cell through caveolae-mediated endocytosis were primarily distributed mainly in the perinuclear region without lysosome trafficking. The shift among clathrin- and caveolae-mediated endocytosis significantly impacted the imaging agent's deterioration. The clathrin-mediated endocytosis directs the imaging agent to the acidic endosomal/lysosomal compartments. The caveolae-mediated endocytosis directs the particles to the nucleus, the endoplasmic reticulum, and the Golgi bodies. In this case, caveolae-mediated transport has led to a more efficient mode of perinuclear trafficking. Subsequently, exocytosis was implemented as the multivalency increased further (40 ligands per particle).

Many researchers have studied the impact of modifying the folate ligand density on ovalbumin-decorated polystyrene nanoparticles' surface and their internalization in a separate study. They concluded that the particle's absorption 
improved to a saturation point with increasing ligand density. Furthermore, they studied with two separate endosome inhibitors, i.e., chlorpromazine for clathrin-mediated endocytosis and filipine for caveolae-mediated endocytosis. The particles with higher folate density may have been more influenced by filipine than those with lower folate concentrations were much more influenced by chlorpromazine. It is suggested that a change from clathrin to caveolae mediated the internalization of nanoparticles as ligand density increased [151].

In recent research, to assess the impact of nanoparticle ligand valency and receptor density on cellular internalization, researchers have performed cell uptake studies on ErbB2-targeting, EC1 surface-functionalized micelles at five ligand valencies and 11 receptor densities [152]. They have reported that receptor density to ligand valency ratio of 0.7-4.5 and a minimum of 1.6 linkages between receptor and ligands are required to initiate cellular internalization. In the cell-uptake profile, lower and upper receptor density limits indicated a norm to divide patients with breast cancer into ErbB2-low, ErbB2-medium, and ErbB2-high. Each category of patients responded differently in response to multivalent nanoparticles (Fig. 8A). Authors have reported that the increment of ligand valence to 40 -valent ErbB2 targeting peptides for a $20-\mathrm{nm}$ radius nanoparticle at the ErbB2-medium and ErbB2-high levels potentiated the cellular internalization (Fig. 8B), suggesting the use of nanocarriers with a higher valency of targeting moiety for effective drug delivery [152].

Each of these examples tends to demonstrate valencydependent shifts between different endocytic pathways. It appears to contribute to the previous discussion about how avidity and target specificity can be related to some optimum value of valency, based on the strength of the multivalent binding event and the receptor's density at a particular cell surface. The studies so far have demonstrated that the multivalent effect seems to have a much-reaching influence on ligands' bioavailability. Specifications such as (1) the concentration of the local receptor, (2) the form of the linker (flexible or rigid), (3) the distance between the ligand and the receptor, and (4) the valence of the ligand all relate in distinctive perspectives to multivalent activity [1].

\section{Optimization is the key: more is not always better}

Throughout this review, we have tried to illustrate the critical importance of designing and developing multivalent nanoconstructs. Considering that more is not always the best option, multivalent ligand grafted nanoconstructs can also result in adverse events if the magnitude of multivalently grafted ligands is not controlled or appropriately optimized. Some penalties may have to be paid off when the surplus number of ligands are grafted on the surface of nanoconstructs. The associated penalties can be categorized as (a) loss of stealth property of nanoconstructs, as a consequence of low surface presentation of stealth molecules such as PEG at the surplus level of ligands, which consequently can result in opsonization and RES clearance of the multivalent nanoconstruct from the systemic circulation; (b) grafting a surplus amount of ligand onto a nanoconstruct may lead to increase in particle diameter, which can hamper its accumulation inside the target cell; and (c) decreased diffusion coefficient of the multivalent nanoconstruct inside the target cell. In this section, we will discuss the downside associated with the concept of multivalency and emphasize more on the discussion that when this concept becomes too much to provide a favorable outcome [68]. Hong et al. [153], in a study, utilized dendrimer as a multivalent nanoconstruct, which is functionalized with varying amounts of folic acid

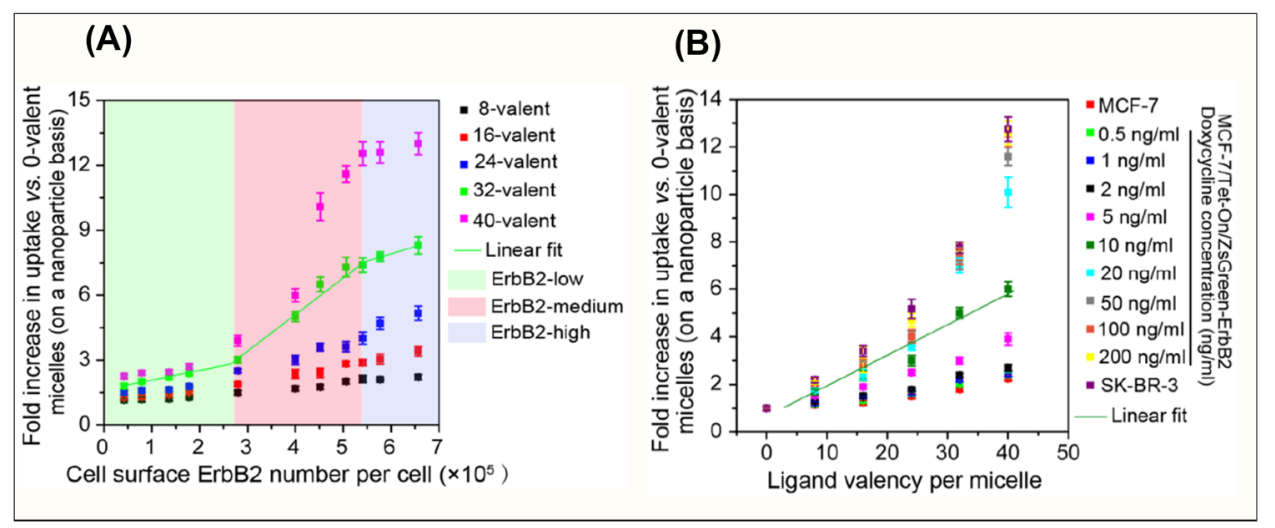

Fig. 8 Cellular internalization of EC1 surface-modified micelles as a function of ErbB2 receptor density and EC1 ligand valency. A Cellular internalization of micelles as a function of receptor density for different ligand valencies. B Cellular internalization of micelles as a function of ligand valency for MCF-7/Tet-On/ZsGreen-ErbB2 cells at varying doxycycline concentrations (reused from Wang et al. [152] permission with American Chemical Society, Copyright 2020) 
ligand ( $n=1-14, n$ : number of ligands per particle), it has been reported by the authors that optimally $n=5$ (plateau) is sufficient for both cell adhesion and overall association constant as quantified by flow cytometry. According to Wan et al. [154], a 100-fold increase in acetylcholine receptor inhibition was achieved in a $n=2$ or divalent alpha conotoxin dendrimer compared to its monovalent counterpart. However, increasing the valency from $n=2$ to $n=4$ resulted in no additional inhibition. In another study, Gu et al. [155] prepared nanoparticles, grafted with PEG and aptamer as a ligand (targeting prostate-specific membrane antigen). Authors have effectively controlled the amount of PEG and aptamer to be surface grafted onto the nanoparticle by altering the ratio of PLGA-b-PEG and PLGA-b-PEGbAPTAMER triblock copolymer. The authors demonstrated that raising the aptamer ratio on the nanoparticle improves the rate of endocytosis by the cells until a plateau phase is achieved. However, with high aptamer surface density, in vivo tumor targeting decreased, accumulation within the liver and spleen increased. The authors explained the significant difference between in vivo and in vitro results because a higher ratio of aptamer masks the PEG grafting on the surface of nanoparticles and decreases in vivo stealth charter of nanoparticles considerable RES (reticuloendothelial system) uptake. Keeping in mind that more is always not better, excellent work by Hortiguela et al. [156] demonstrates that design and optimization are of utmost priority when considering multivalent nanoconstructs as a drug delivery option. Authors have reported a self-assembled nanostructured surface (known as nanopattern) capable of initiating multivalent interactions between surface grafted ephrinB 1 ligands and EphB2 receptors. The findings demonstrate that arbitrarily dispersed surface-bound ligands were insufficient to promote receptor clustering properly. In contrast, when the ligands were nanopatterned onto substrates, they successfully generated a robust receptor oligomerization. Such kind of surface ligand grafting enhanced the clustering effectiveness of conventional ligand delivery methods by requiring ninefold less ligand surface coverage and quicker receptor clustering kinetics than standard cross-linked ligands.

Kappel et al. [157] recently investigated the extent to which surface grafting of nanoparticles with antibodytargeting can cause off-target accumulation, for instance, in the liver, when nanoparticles are administered intravenously. To demonstrate this, the authors engineered polysarcosine polymer brushes with varying average numbers ( $n=2,6$, and 12) of antibodies specific to dendritic cell surface receptors (DEC205). Authors have shown that an average of 2 antibodies per nanoparticle is sufficient to target lymphoid organs specifically. However, nanoparticles functionalized with more than two antibodies get dumped in the liver due to their recognition by complement receptors. It is important to note here that if the antibody numbers per nanoparticle are kept low, the capture of the nanoparticle by the liver sinusoidal cells can be prevented. These results are alarming, since they indicate that in order to target drug delivery, we often use more than the required amount of targeting moiety for selective targeting. Furthermore, these results imply that when designing targeted multivalent nanoparticles, the ratio of targeting moieties per nanoparticle should be optimized for maximum targeting.

\section{Conclusion and future perspectives}

Following long-standing, groundbreaking work on multivalent medications, hardly any prominent pharmaceutical firm has actively engaged in developing multivalent drug delivery therapeutics. The explanation is undoubtedly the intense emphasis on "small molecules" and now on "biologicals." The multivalency principle has been intensively researched in many different systems since the seminal study of biologically applicable multivalent structures by Mammen and coworkers [3] in 1998. Multivalent ligands are more beneficial than monovalent ones because of their tremendous binding power across stable, defined spacers, and these ligands are increasingly being introduced to pharmaceutically applicable targets. As drug delivery progresses towards site-specific, target-oriented methodologies, multivalent interactions are becoming an effective strategy in designing guided/targeted drug delivery applications. It is crucial when it comes to designing clinical approaches that target cellular receptors. This review has demonstrated the need for a better theoretical understanding of the multivalent effect of what happens to the receptor-ligand complex once it is established. The research itself would also gain further from experiments that look past the initial ligand-receptor interaction. It would disclose a more massive effect of multivalent interactions on ligands' bioavailability and pave new dimensions towards designing and developing effective multivalent drug delivery nanoconstructs.

Author contribution $\mathrm{AKB}$ and $\mathrm{TH}$ equally contributed in this study. $\mathrm{AKB}, \mathrm{TH}$ and $\mathrm{VS}$ prepared the preliminary draft for the manuscript. $\mathrm{AKB}, \mathrm{TH}$, and $\mathrm{RT}$ contributed to the writing of the manuscript. $\mathrm{TH}$, $\mathrm{AKB}$ and VS jointly developed the structure and arguments for the paper. TH and VS made critical revisions and approved the final version of the paper. All authors reviewed and approved the final manuscript.

Availability of data and materials Data and material are available upon request.

\section{Declarations}

Ethics approval and consent to participate NA.

Consent for publication NA.

Competing interests The authors declare no competing interests. 


\section{References}

1. Fasting C, Schalley CA, Weber M, et al. Multivalency as a chemical organization and action principle. Angew Chem Int Edit. 2012;51(42):10472-98.

2. Kiessling LL, Gestwicki JE, Strong LE. Synthetic multivalent ligands as probes of signal transduction. Angew Chem Int. 2006;45(15):2348-68.

3. Mammen M, Choi SK, Whitesides GM. Polyvalent interactions in biological systems: implications for design and use of multivalent ligands and inhibitors. Angew Chem Int. 1998;37(20):2754-94.

4. Hunter CA, Anderson HL. What is cooperativity? Angew Chem Int Ed Engl. 2009;48(41):7488-99.

5. Mannhold R, Kubinyi H, Folkers G. Fragment-based approaches in drug discovery, vol. 34. John Wiley \& Sons; 2006.

6. Schweitzer-Stenner R, Licht A, Pecht I. Dimerization kinetics of the IgE-class antibodies by divalent haptens I The Fab-hapten interactions. Biophys J. 1992;63(2):551-62.

7. Schweitzer-Stenner R, Licht A, Luscher I, et al. Oligomerization and ring closure of immunoglobulin $\mathrm{E}$ class antibodies by divalent haptens. Biochemistry. 1987;26(12):3602-12.

8. Ewers H, Romer W, Smith AE, et al. GM1 structure determines SV40-induced membrane invagination and infection. Nat Cell Biol. 2010;12(1):11-8.

9. Ling H, Boodhoo A, Hazes B, et al. Structure of the shiga-like toxin I B-pentamer complexed with an analogue of its receptor Gb3. Biochemistry. 1998;37(7):1777-88.

10. Kitov PI, Paszkiewicz E, Sadowska JM, et al. Impact of the nature and size of the polymeric backbone on the ability of heterobifunctional ligands to mediate shiga toxin and serum amyloid p component ternary complex formation. Toxins (Basel). 2011;3(9):1065-88.

11. Stone E, Hirama T, Chen W, et al. A novel pentamer versus pentamer approach to generating neutralizers of verotoxin 1. Mol Immunol. 2007;44(9):2487-91.

12. Mowery P, Yang ZQ, Gordon EJ, et al. Synthetic glycoprotein mimics inhibit L-selectin-mediated rolling and promote L-selectin shedding. Chem Biol. 2004;11(5):725-32.

13. Pieters RJ. Maximising multivalency effects in protein-carbohydrate interactions. Org Biomol Chem. 2009;7(10):2013-25.

14. Chabre YM, Roy R. Recent trends in glycodendrimer syntheses and applications. Curr Top Med Chem. 2008;8(14):1237-85.

15. Chabre YM, Roy R. Design and creativity in synthesis of multivalent neoglycoconjugates. Advances in carbohydrate chemistry and biochemistry, vol. 63. Elsevier; 2010. p. 165-393.

16. Woller EK, Walter ED, Morgan JR, et al. Altering the strength of lectin binding interactions and controlling the amount of lectin clustering using mannose/hydroxyl-functionalized dendrimers. J Am Chem Soc. 2003;125(29):8820-6.

17. Munoz-Torrero D. Exploiting multivalency in drug design. Curr Pharm Des. 2009;15(6):585-6.

18. Riedel T, Ghasparian A, Moehle K, et al. Synthetic virus-like particles and conformationally constrained peptidomimetics in vaccine design. ChemBioChem. 2011;12(18):2829-36.

19. Luo XM, Lei MY, Feidi RA, et al. Dimeric $2 \mathrm{G} 12$ as a potent protection against HIV-1. PLoS Pathogens. 2010;6(12):e1001225.

20. Staropoli I, Chanel C, Girard M, et al. Processing, stability, and receptor binding properties of oligomeric envelope glycoprotein from a primary HIV-1 isolate. J Biol Chem. 2000;275(45):35137-45.

21. Chittasupho C. Multivalent ligand: design principle for targeted therapeutic delivery approach. Ther Deliv. 2012;3(10):1171-87.

22. Dower SK, Titus JA, DeLisi C, et al. Mechanism of binding of multivalent immune complexes to $\mathrm{Fc}$ receptors 2 Kinetics of binding. Biochemistry. 1981;20(22):6335-40.
23. DeFrees SA, Phillips L, Guo L, et al. Sialyl Lewis x liposomes as a multivalent ligand and inhibitor of E-selectin mediated cellular adhesion. J Am Chem Soc. 1996;118(26):6101-4.

24. Kiessling LL, Gestwicki JE, Strong LE. Synthetic multivalent ligands in the exploration of cell-surface interactions. Curr Opin Chem Biol. 2000;4(6):696-703.

25. Papp I, Sieben C, Sisson AL, et al. Inhibition of influenza virus activity by multivalent glycoarchitectures with matched sizes. ChemBioChem. 2011;12(6):887-95.

26. Sykes EA, Chen J, Zheng G, et al. Investigating the impact of nanoparticle size on active and passive tumor targeting efficiency. ACS Nano. 2014;8(6):5696-706.

27. Nasr K, Pannier N, Frangioni JV, et al. Rigid multivalent scaffolds based on adamantane. J Org Chem. 2008;73(3):1056-60.

28. Adeli M, Kalantari M, Zarnega Z, et al. Cyclodextrin-based dendritic supramolecules; new multivalent nanocarriers. Rsc Adv. 2012;2(7):2756-8.

29. Sansone F, Baldini L, Casnati A, et al. Calixarenes: from biomimetic receptors to multivalent ligands for biomolecular recognition. New J Chem. 2010;34(12):2715-28.

30. Hlavacek WS, Posner RG, Perelson AS. Steric effects on multivalent ligand-receptor binding: exclusion of ligand sites by bound cell surface receptors. Biophys J. 1999;76(6):3031-43.

31. Crothers DM, Metzger $\mathrm{H}$. The influence of polyvalency on the binding properties of antibodies. Immunochemistry. 1972;9(3):341-57.

32. Cremers GAO, Rosier B, Meijs A, et al. Determinants of ligandfunctionalized DNA nanostructure-cell interactions. J Am Chem Soc. 2021;143(27):10131-42.

33. Choo P, Liu T, Odom TW. Nanoparticle shape determines dynamics of targeting nanoconstructs on cell membranes. J Am Chem Soc. 2021;143(12):4550-5.

34. Cuesta ÁM, Sainz-Pastor N, Bonet J, et al. Multivalent antibodies: when design surpasses evolution. Trends Biotechnol. 2010;28(7):355-62.

35. Lin C-H, Shen G-J, Garcia-Junceda E, et al. Enzymic synthesis and regeneration of 3'- phosphoadenosine 5'-phosphosulfate (PAPS) for regioselective sulfation of oligosaccharides. J Am Chem Soc. 1995;117(30):8031-2.

36. Kiessling LL, Strong LE, Gestwicki JE. Principles for multivalent ligand design. Annu Rep Med Chem. 2000;35:321-30.

37. Cairo CW, Gestwicki JE, Kanai M, et al. Control of multivalent interactions by binding epitope density. J Am Chem Soc. 2002;124(8):1615-9.

38. Kiessling LL, Strong LE. Bioactive polymers. In: Fürstner A, editor. Alkene metathesis in organic synthesis, vol. 1. Berlin Heidelberg: Springer; 1998. p. 199-231.

39. Maynard HD, Okada SY, Grubbs RH. Inhibition of cell adhesion to fibronectin by oligopeptide-substituted polynorbornenes. J Am Chem Soc. 2001;123(7):1275-9.

40. Trnka TM, Grubbs RH. The development of L2X2Ru CHR olefin metathesis catalysts: an organometallic success story. Acc Chem Res. 2001;34(1):18-29.

41. Lynn DM, Mohr B, Grubbs RH. Living ring-opening metathesis polymerization in water. Acc Chem Res. 1998;120(7):1627-8.

42. Kanai M, Mortell KH, Kiessling LL. Varying the size of multivalent ligands: the dependence of concanavalin A binding on neoglycopolymer length. J Am Chem Soc. 1997;119(41):9931-2.

43. Iyer S, Rele S, Grasa G, et al. Synthesis of a hyaluronan neoglycopolymer by ring-opening metathesis polymerization. Chem Commun (Camb). 2003;13:1518-9.

44. Arimoto H, Nishimura K, Hayakawa I, et al. Multi-valent polymer of vancomycin: enhanced antibacterial activity against VRE. Chem Commun. 1999;15:1361-2. 
45. Gestwicki JE, Kiessling LL. Inter-receptor communication through arrays of bacterial chemoreceptors. Nature. 2002;415(6867):81-4.

46. Gordon EJ, Sanders WJ, Kiessling LL. Synthetic ligands point to cell surface strategies. Nature. 1998;392(6671):30-1.

47. Cochran JR, Aivazian D, Cameron TO, et al. Receptor clustering and transmembrane signaling in $\mathrm{T}$ cells. Trends Biochem Sci. 2001;26(5):304-10.

48. Cochran JR, Stern LJ. A diverse set of oligomeric class II MHCpeptide complexes for probing T-cell receptor interactions. Chem Biol. 2000;7(9):683-96.

49. Sulzer B, Perelson AS. Immunons revisited: binding of multivalent antigens to B cells. Mol Immunol. 1997;34(1):63-74.

50. Ragupathi G, Howard L, Cappello S, et al. Vaccines prepared with sialyl-Tn and sialyl-Tn trimers using the 4-(4-maleimidomethyl) cyclohexane-1-carboxyl hydrazide linker group result in optimal antibody titers against ovine submaxillary mucin and sialyl-Tnpositive tumor cells. Cancer Immunol Immunother. 1999;48(1):1-8.

51. Dintzis RZ, Okajima M, Middleton M, et al. The immunogenicity of soluble haptenated polymers is determined by molecular mass and hapten valence. J Immunol. 1989;143(4):1239-44.

52. Radford DC, Yang J, Doan MC, et al. Multivalent HER2-binding polymer conjugates facilitate rapid endocytosis and enhance intracellular drug delivery. J Control Release. 2020;319:285-99.

53. Krishnamurthy VM, Semetey V, Bracher PJ, et al. Dependence of effective molarity on linker length for an intramolecular proteinligand system. J Am Chem Soc. 2007;129(5):1312-20.

54. Shewmake TA, Solis FJ, Gillies RJ, et al. Effects of linker length and flexibility on multivalent targeting. Biomacromol. 2008;9(11):3057-64.

55. Hsu CL, Chang HT, Chen CT, et al. Highly efficient control of thrombin activity by multivalent nanoparticles. Chemistry (Easton). 2011;17(39):10994-1000.

56. Lundquist JJ, Toone EJ. The cluster glycoside effect. Chem Rev. 2002;102(2):555-78.

57. Kitov PI, Sadowska JM, Mulvey G, et al. Shiga-like toxins are neutralized by tailored multivalent carbohydrate ligands. Nature. 2000;403(6770):669-72.

58. Merritt EA, Zhang Z, Pickens JC, et al. Characterization and crystal structure of a high-affinity pentavalent receptor-binding inhibitor for cholera toxin and E. coli heat-labile enterotoxin. J Am Chem Soc. 2002;124(30):8818-24.

59. Krishnamurthy VM, Estroff LA, Whitesides GM. Multivalency in ligand design. Fragm Based Approaches Drug Discov. 2006;34:11-53.

60. Mallikaratchy PR, Ruggiero A, Gardner JR, et al. A multivalent DNA aptamer specific for the B-cell receptor on human lymphoma and leukemia. Nucleic Acids Res. 2011;39(6):2458-69.

61. Shiokawa T, Hattori Y, Kawano K, et al. Effect of polyethylene glycol linker chain length of folate-linked microemulsions loading aclacinomycin A on targeting ability and antitumor effect in vitro and in vivo. Clin Cancer Res. 2005;11(5):2018-25.

62. Arnold M, Cavalcanti-Adam EA, Glass R, et al. Activation of integrin function by nanopatterned adhesive interfaces. ChemPhysChem. 2004;5(3):383-8.

63. Rosca EV, Stukel JM, Gillies RJ, et al. Specificity and mobility of biomacromolecular, multivalent constructs for cellular targeting. Biomacromol. 2007;8(12):3830-5.

64. Gestwicki JE, Cairo CW, Strong LE, et al. Influencing receptorligand binding mechanisms with multivalent ligand architecture. J Am Chem Soc. 2002;124(50):14922-33.

65. Gestwicki JE, Strong LE, Cairo CW, et al. Cell aggregation by scaffolded receptor clusters. Chem Biol. 2002;9(2):163-9.

66. Mann DA, Kanai M, Maly DJ, et al. Probing low affinity and multivalent interactions with surface plasmon resonance: ligands for concanavalin A. J Am Chem Soc. 1998;120(41):10575-82.
67. Bandyopadhyay A, Fine RL, Demento S, et al. The impact of nanoparticle ligand density on dendritic-cell targeted vaccines. Biomaterials. 2011;32(11):3094-105.

68. Alkilany AM, Zhu L, Weller H, et al. Ligand density on nanoparticles: a parameter with critical impact on nanomedicine. Adv Drug Deliv Rev. 2019;143:22-36.

69. Robinson A, Fang JM, Chou PT, et al. Probing lectin and sperm with carbohydrate-modified quantum dots. ChemBioChem. 2005;6(10):1899-905.

70. de Mol NJ, Dekker FJ, Broutin I, et al. Surface plasmon resonance thermodynamic and kinetic analysis as a strategic tool in drug design. Distinct ways for phosphopeptides to plug into Src-and Grb2 SH2 domains. J Med Chem. 2005;48(3):753-63.

71. Perlmutter-Hayman B. Cooperative binding to macromolecules. A formal approach Acc Chem Res. 1986;19(3):90-6.

72. Ercolani G. Assessment of cooperativity in self-assembly. J Am Chem Soc. 2003;125(51):16097-103.

73. Huskens J, Mulder A, Auletta T, et al. A model for describing the thermodynamics of multivalent host-guest interactions at interfaces. J Am Chem Soc. 2004;126(21):6784-97.

74. Wolfenden ML, Cloninger MJ. Mannose/glucose-functionalized dendrimers to investigate the predictable tunability of multivalent interactions. J Am Chem Soc. 2005;127(35):12168-9.

75. Thordarson P. Determining association constants from titration experiments in supramolecular chemistry. Chem Soc Rev. 2011;40(3):1305-23.

76. Gargano JM, Ngo T, Kim JY, et al. Multivalent inhibition of AB5 toxins. J Am Chem Soc. 2001;123(51):12909-10.

77. Kitov PI, Bundle DR. On the nature of the multivalency effect: a thermodynamic model. J Am Chem Soc. 2003;125(52):16271-84.

78. Jacobson H, Stockmayer WH. Intramolecular reaction in polycondensations. I. The theory of linear systems. J Chem Phys. 1950;18(12):1600-6.

79. Mulder A, Huskens J, Reinhoudt DN. Multivalency in supramolecular chemistry and nanofabrication. Org Biomol Chem. 2004;2(23):3409-24.

80. Schmidtchen FP. Isothermal titration calorimetry in supramolecular chemistry. In: Schalley CA, editor. Analytical methods in supramolecular chemistry, vol. 1. John Wiley \& Sons; 2012. p. 55-78.

81. Zhang S, Leem G, Srisombat L-o, et al. Rationally designed ligands that inhibit the aggregation of large gold nanoparticles in solution. J Am Chem Soc. 2008;130(1):113-20.

82. Scheibe C, Bujotzek A, Dernedde J, et al. DNA-programmed spatial screening of carbohydrate-lectin interactions. Chem Sci. 2011;2(4):770-5.

83. Nahshol O, Bronner V, Notcovich A, et al. Parallel kinetic analysis and affinity determination of hundreds of monoclonal antibodies using the ProteOn XPR36. Anal Biochem. 2008;383(1):52-60.

84. Gavutis M, Lata S, Piehler J. Probing 2-dimensional proteinprotein interactions on model membranes. Nat Protoc. 2006;1(4):2091-103.

85. Evan-Salem T, Baruch I, Avram L, et al. Resorcinarenes are hexameric capsules in solution. Proc Natl Acad Sci USA 2006;103(33):12296-300.

86. Yu L, Huang M, Wang PG, et al. Cross-linked surface-grafted glycopolymer for multivalent recognition of lectin. Anal Chem. 2007;79(23):8979-86.

87. Gussin HA, Tomlinson ID, Muni NJ, et al. GABAC receptor binding of quantum-dot conjugates of variable ligand valency. Bioconjug Chem. 2010;21(8):1455-64.

88. Dernedde J, Rausch A, Weinhart M, et al. Dendritic polyglycerol sulfates as multivalent inhibitors of inflammation. Proc Natl Acad Sci. 2010;107(46):19679-84. 
89. Braun P, Nägele B, Wittmann V, et al. Mechanistische Untersuchung multivalenter Kohlenhydrat-Protein-Wechselwirkungen durch EPR-Spektroskopie. Angew Chem. 2011;123(36):8579-82.

90. Voskuhl J, Stuart MC, Ravoo BJ. Sugar-decorated sugar vesicles: lectin-carbohydrate recognition at the surface of cyclodextrin vesicles. Chem Eur J. 2010;16(9):2790-6.

91. Handa H, Gurczynski S, Jackson MP, et al. Immobilization and molecular interactions between bacteriophage and lipopolysaccharide bilayers. Langmuir. 2010;26(14):12095-103.

92. Rao J, Lahiri J, Isaacs L, et al. A trivalent system from vancomycin. d-Ala-d-Ala with higher affinity than avidin - biotin. Science. 1998;280(5364):708-11.

93. Janssen PG, Brankaert NJ, Vila X, et al. ssDNA templated assembly of oligonucleotides and bivalent naphthalene guests. Soft Matter. 2010;6(7):1494-502.

94. Tsai H-C, Imae T. Fabrication of dendrimers toward biological application Progress in molecular biology and translational science, vol. 104. Elsevier; 2011. p. 101-140.

95. Khandare J, Calderon M, Dagia NM, et al. Multifunctional dendritic polymers in nanomedicine: opportunities and challenges. Chem Soc Rev. 2012;41(7):2824-48.

96. Liu X, Liu C, Laurini E, et al. Efficient delivery of sticky siRNA and potent gene silencing in a prostate cancer model using a generation 5 triethanolamine-core PAMAM dendrimer. Mol Pharm. 2012;9(3):470-81.

97. Xin G, Zhao X, Duan X, et al. Antitumor effect of a generation 4 polyamidoamine dendrimer/cyclooxygenase- 2 antisense oligodeoxynucleotide complex on breast cancer in vitro and in vivo. Cancer Biother Radiopharm. 2012;27(1):77-87.

98. Frechet JM, Lochman L, Šmigol V, et al. Reversed-phase highperformance liquid chromatography of functionalized dendritic macromolecules. J Chromatogr A. 1994;667(1-2):284-9.

99. Hawker CJ, Frechet JM. Preparation of polymers with controlled molecular architecture A new convergent approach to dendritic macromolecules. J Am Chem Soc. 1990;112(21):7638-47.

100. Grayson SM, Frechet JM. Convergent dendrons and dendrimers: from synthesis to applications. Chem Rev. 2001;101(12):3819-68.

101. Hawker C, Fréchet JM. A new convergent approach to monodisperse dendritic macromolecules. J Chem Soc Chem Commun. 1990;15:1010-3.

102. Tomalia DA. Dendrimers as multi-purpose nanodevices for oncology drug delivery and diagnostic imaging. Nanomed Nanotechnol Biol Med. 2006;4(2):309.

103. Esfand R, Tomalia DA. Poly (amidoamine)(PAMAM) dendrimers: from biomimicry to drug delivery and biomedical applications. Drug Discov Today. 2001;6(8):427-36.

104. Menjoge AR, Kannan RM, Tomalia DA. Dendrimer-based drug and imaging conjugates: design considerations for nanomedical applications. Drug Discov Today. 2010;15(5-6):171-85.

105. Newkome GR, He E, Moorefield CN. Suprasupermolecules with novel properties: metallodendrimers. Chem Rev. 1999;99(7):1689-746.

106. Walter MV, Malkoch M. Simplifying the synthesis of dendrimers: accelerated approaches. Chem Soc Rev. 2012;41(13):4593-609.

107. Fournier D, Hoogenboom R, Schubert US. Clicking polymers: a straightforward approach to novel macromolecular architectures. Chem Soc Rev. 2007;36(8):1369-80.

108. Ihre H, Hult A, Fréchet JM, et al. Double-stage convergent approach for the synthesis of functionalized dendritic aliphatic polyesters based on 2,2-bis (hydroxymethyl) propionic acid. Macromolecules. 1998;31(13):4061-8.

109. Burai R, Chatwichien J, McNaughton BR. A programmable "build-couple" approach to the synthesis of heterofunctionalized polyvalent molecules. Org Biomol Chem. 2011;9(14):5056-8.

110. Holub JM, Garabedian MJ, Kirshenbaum K. Peptoids on steroids: precise multivalent estradiol-peptidomimetic conjugates generated via azide-alkyne [3+2] cycloaddition reactions. QSAR Comb Sci. 2007;26(11-12):1175-80.

111. Tomalia DA, Christensen JB, Boas U. Dendrimers, dendrons, and dendritic polymers: discovery applications and the future. Cambridge University Press; 2012.

112. Christensen JB, Tomalia DA. Dendrimers as quantized nanomodules in the nanotechnology field. J. Hoboken, NJ: Wiley \& Sons; 2012.

113. Bezouška K. Design, functional evaluation and biomedical applications of carbohydrate dendrimers (glycodendrimers). Rev Mol Biotechnol. 2002;90(3-4):269-90.

114. Wang S-K, Liang P-H, Astronomo RD, et al. Targeting the carbohydrates on HIV-1: interaction of oligomannose dendrons with human monoclonal antibody 2G12 and DC-SIGN. Proc Natl Acad Sci. 2008;105(10):3690-5.

115. Graf N, Bielenberg DR, Kolishetti N, et al. $\alpha \mathrm{V} \beta 3$ integrintargeted PLGA-PEG nanoparticles for enhanced anti-tumor efficacy of a Pt (IV) prodrug. ACS Nano. 2012;6(5):4530-9.

116. Montet X, Funovics M, Montet-Abou K, et al. Multivalent effects of RGD peptides obtained by nanoparticle display. J Med Chem. 2006;49(20):6087-93.

117. Hussain S, Joo J, Kang J, et al. Antibiotic-loaded nanoparticles targeted to the site of infection enhance antibacterial efficacy. Nat Biomed Eng. 2018;2(2):95-103.

118. Kiziltepe T, Ashley J, Stefanick J, et al. Rationally engineered nanoparticles target multiple myeloma cells overcome celladhesion-mediated drug resistance and show enhanced efficacy in vivo. Blood Cancer J. 2012;2(4):e64-e64.

119. Zhang Y, Cheng M, Cao J, et al. Multivalent nanoparticles for personalized theranostics based on tumor receptor distribution behavior. Nanoscale. 2019;11(11):5005-13.

120. Abstiens K, Gregoritza M, Goepferich AM. Ligand density and linker length are critical factors for multivalent nanoparticle-receptor interactions. ACS Appl Mater Interfaces. 2019;11(1):1311-20.

121. Maslanka Figueroa S, Fleischmann D, Beck S, et al. The effect of ligand mobility on the cellular interaction of multivalent nanoparticles. Macromol Biosci. 2020;20(4):e1900427.

122. Karimi F, O'Connor AJ, Qiao GG, et al. Integrin clustering matters: a review of biomaterials functionalized with multivalent integrin-binding ligands to improve cell adhesion migration differentiation angiogenesis and biomedical device integration. Adv Healthc Mater. 2018;7(12):e1701324.

123. Govindan SV, Goldenberg DM. Designing immunoconjugates for cancer therapy. Expert Opin Biol Ther. 2012;12(7):873-90.

124. Litvak-Greenfeld D, Benhar I. Risks and untoward toxicities of antibody-based immunoconjugates. Adv Drug Del Rev. 2012;64(15):1782-99.

125. Pasquetto MV, Vecchia L, Covini D, et al. Targeted drug delivery using immunoconjugates: principles and applications. J Immunother. 2011;34(9):611-28.

126. Ogura M, Hatake K, Ando K, et al. Phase I study of anti-CD 22 immunoconjugate inotuzumab ozogamicin plus rituximab in relapsed/refractory B-cell non-H odgkin lymphoma. Cancer Sci. 2012;103(5):933-8.

127. Johnson RN, Kopečková P, Kopecek J. Synthesis and evaluation of multivalent branched HPMA copolymer-Fab' conjugates targeted to the B-cell antigen CD20. Bioconjug Chem. 2009;20(1):129-37.

128. Rizvi S, Allen BJ, Lee CS, et al. Orthotopic administration of 213Bi-bevacizumab inhibits progression of PC3 xenografts in the prostate. Immunotherapy. 2012;4(5):549-54.

129. Sharkey RM, Rossi EA, Chang C-H, et al. Improved cancer therapy and molecular imaging with multivalent, multispecific antibodies. Cancer Biother Radiopharm. 2010;25(1):1-12. 
130. Cuesta AM, Sanchez-Martin D, Blanco-Toribio A, et al. Improved stability of multivalent antibodies containing the human collagen XV trimerization domain. MAbs. 2012;4(2):226-32.

131. Dillman RO. Radiolabeled anti-CD20 monoclonal antibodies for the treatment of B-cell lymphoma. J Clin Oncol. 2002;20(16):3545-57.

132. Delalat B, Sheppard VC, Ghaemi SR, et al. Targeted drug delivery using genetically engineered diatom biosilica. Nat Commun. 2015;6(1):1-11.

133. Rujas E, Kucharska I, Tan YZ, et al. Multivalency transforms SARS-CoV-2 antibodies into ultrapotent neutralizers. Nat Commun. 2021;12(1):1-12.

134. Kaltgrad E, Sen Gupta S, Punna S, et al. Anti-carbohydrate antibodies elicited by polyvalent display on a viral scaffold. ChemBioChem. 2007;8(12):1455-62.

135. Liepold LO, Abedin MJ, Buckhouse ED, et al. Supramolecular protein cage composite MR contrast agents with extremely efficient relaxivity properties. Nano Lett. 2009;9(12):4520-6.

136. Kim B-S, Hong D-J, Bae J, et al. Controlled self-assembly of carbohydrate conjugate rod-coil amphiphiles for supramolecular multivalent ligands. J Am Chem Soc. 2005;127(46):16333-7.

137. Simnick AJ, Valencia CA, Liu R, et al. Morphing low-affinity ligands into high-avidity nanoparticles by thermally triggered self-assembly of a genetically encoded polymer. ACS Nano. 2010;4(4):2217-27.

138. Xu H, Medina-Sánchez M, Magdanz V, et al. Spermhybrid micromotor for targeted drug delivery. ACS Nano. 2018;12(1):327-37.

139. Sharma S, Kotamraju VR, Mölder T, et al. Tumor-penetrating nanosystem strongly suppresses breast tumor growth. Nano Lett. 2017;17(3):1356-64.

140. Agemy L, Friedmann-Morvinski D, Kotamraju VR, et al. Targeted nanoparticle enhanced proapoptotic peptide as potential therapy for glioblastoma. Proc Natl Acad Sci. 2011;108(42):17450-5.

141. Aluri SR, Shi P, Gustafson JA, et al. A hybrid protein-polymer nanoworm potentiates apoptosis better than a monoclonal antibody. ACS Nano. 2014;8(3):2064-76.

142. Lee C, Peddi S, Anderson C, et al. Adaptable antibody nanoworms designed for non-Hodgkin lymphoma. Biomaterials. 2020;262:120338.

143. Luo H, Yang J, Jin H, et al. Tetrameric far-red fluorescent protein as a scaffold to assemble an octavalent peptide nanoprobe for enhanced tumor targeting and intracellular uptake in vivo. FASEB J. 2011;25(6):1865-73.

144. Kostiainen MA, Szilvay GR, Lehtinen J, et al. Precisely defined protein-polymer conjugates: construction of synthetic DNA binding domains on proteins by using multivalent dendrons. ACS Nano. 2007;1(2):103-13.
145. Li W, Yang X, He L, et al. Self-assembled DNA nanocentipede as multivalent drug carrier for targeted delivery. ACS Appl Mater Interfaces. 2016;8(39):25733-40.

146. Li S, Jiang Q, Liu S, et al. A DNA nanorobot functions as a cancer therapeutic in response to a molecular trigger in vivo. Nat Biotechnol. 2018;36(3):258.

147. Caldorera-Moore M, Guimard N, Shi L, et al. Designer nanoparticles: incorporating size, shape and triggered release into nanoscale drug carriers. Expert Opin Drug Deliv. 2010;7(4):479-95.

148. Sahay G, Alakhova DY, Kabanov AV. Endocytosis of nanomedicines. J Control Release. 2010;145(3):182-95.

149. Dalal C, Saha A, Jana NR. Nanoparticle multivalency directed shifting of cellular uptake mechanism. J Phys Chem C. 2016;120(12):6778-86.

150. Dalal C, Jana NR. Multivalency effect of TAT-peptidefunctionalized nanoparticle in cellular endocytosis and subcellular trafficking. J Phys Chem B. 2017;121(14):2942-51.

151. Moradi E, Vllasaliu D, Garnett M, et al. Ligand density and clustering effects on endocytosis of folate modified nanoparticles. Rsc Adv. 2012;2(7):3025-33.

152. Wang J, Min J, Eghtesadi SA, et al. Quantitative study of the interaction of multivalent ligand-modified nanoparticles with breast cancer cells with tunable receptor density. ACS Nano. 2020;14(1):372-83.

153. Hong S, Leroueil PR, Majoros IJ, et al. The binding avidity of a nanoparticle-based multivalent targeted drug delivery platform. Chem Biol. 2007;14(1):107-15.

154. Wan J, Huang JX, Vetter I, et al. alpha-Conotoxin dendrimers have enhanced potency and selectivity for homomeric nicotinic acetylcholine receptors. J Am Chem Soc. 2015;137(9):3209-12.

155. Gu F, Zhang L, Teply BA, et al. Precise engineering of targeted nanoparticles by using self-assembled biointegrated block copolymers. Proc Natl Acad Sci USA. 2008;105(7):2586-91.

156. Hortiguela V, Larranaga E, Cutrale F, et al. Nanopatterns of surface-bound EphrinB1 produce multivalent ligand-receptor interactions that tune EphB2 receptor clustering. Nano Lett. 2018;18(1):629-37.

157. Kappel C, Seidl C, Medina-Montano C, et al. Density of conjugated antibody determines the extent of fc receptor dependent capture of nanoparticles by liver sinusoidal endothelial cells. ACS Nano. 2021;15(9):15191-209.

Publisher's Note Springer Nature remains neutral with regard to jurisdictional claims in published maps and institutional affiliations. 